\title{
Misiurewicz parameters and dynamical stability of polynomial-like maps of large topological degree
}

\author{
Fabrizio Bianchi ${ }^{1}$
}

Received: 21 February 2017 / Revised: 10 December 2017 / Published online: 19 January 2018

(C) The Author(s) 2018. This article is an open access publication

\begin{abstract}
Given a family of polynomial-like maps of large topological degree, we relate the presence of Misiurewicz parameters to a growth condition for the volume of the iterates of the critical set. This generalizes to higher dimensions the well-known equivalence between stability and normality of the critical orbits in dimension one. We also introduce a notion of holomorphic motion of asymptotically all repelling cycles and prove its equivalence with other notions of stability. Our results allow us to generalize the theory of stability and bifurcation developed by Berteloot, Dupont and the author for the family of all endomorphisms of $\mathbb{P}^{k}$ of a given degree to any arbitrary family of endomorphisms of $\mathbb{P}^{k}$ or polynomial-like maps of large topological degree.
\end{abstract}

Mathematics Subject Classification 32H50 - 32U40 - 37F45 - 37F50 · 37H15

\section{Introduction and results}

The goal of this paper is to study the dynamical stability within arbitrary families of polynomial-like maps of large topological degree and endomorphisms of $\mathbb{P}^{k}$. We aim to generalize to this setting the theory developed in [5] for the family of all endomorphisms of $\mathbb{P}^{k}$ of a given degree. This requires a quite different approach with respect to that situation. In particular, our main result relates bifurcations in such families to the growth of the volume of the critical set under iteration. This generalizes

Communicated by Ngaiming Mok.

$凶$ Fabrizio Bianchi

f.bianchi@imperial.ac.uk

1 Imperial College, South Kensington Campus, London SW7 2AZ, UK 
the one-dimensional equivalence between dynamical stability and normality of the critical orbits.

The study of dynamical stability within families of holomorphic dynamical systems $f_{\lambda}$ goes back to the 80s, when Lyubich [22] and Mañé-Sad-Sullivan [23] independently set the foundations of the study of holomorphic families of rational maps in dimension one. They proved that various natural definitions of stability (like the holomorphic motion of the repelling cycles, or of the Julia set, or the Hausdorff continuity of the latter) are actually equivalent and that the stable behaviour occurs on an open and dense subset of the parameter space.

In 2000, De Marco $[10,11]$ proved a formula relating the Lyapunov function $L\left(f_{\lambda}\right)$ of a rational map to the critical dynamics of the family. It turns out that the canonical closed and positive $(1,1)$-current $d d_{\lambda}^{c} L$ on the parameter space is exactly supported on the bifurcation locus (the observation that the bifurcation measure for the family $z^{2}+c$ is equal to the harmonic measure for the Mandelbrot set is due to N. Sibony, see [27]). This allowed for the start of a measure-theoretic study of bifurcations.

In recent years, there has been growing interest in trying to generalize the theory by Lyubich, Mañé-Sad-Sullivan and De Marco to higher dimensions. The works by Berger, Dujardin and Lyubich [2,16] are dedicated to the stability of Hénon maps, while the work [5] is concerned with the family of all endomorphisms of $\mathbb{P}^{k}$ of a given degree. This is the higher-dimensional analogue of rational maps, and the bifurcation locus in this setting turns out to coincide with the support of $d d^{c} L$, as in dimension one. The generalization to this setting of De Marco's formula due to Bassanelli-Berteloot [1] and the existence of potentials for the measures of maximal entropy are crucial in this study. The main goal of this paper is to generalize the results of [5] to the setting of polynomial like maps of large topological degree, and at the same time to extend that theory to an arbitrary family of endomorphisms of $\mathbb{P}^{k}$. We basically have to face two difficulties. The first concerns the relation between global and critical stabilities and is related to the absence of potentials. This will be overcome by a more geometric approach, with the additional value of giving a more quantitative insight on bifurcations. The second deals with the bifurcations of repelling cycles and is related to the absence of an algebraic structure for the families we consider. As a consequence of our approach, we will also be able to establish an extended version of the theory of [5] to arbitrary families of endomorphisms of $\mathbb{P}^{k}$, a question left widely open in that paper.

Polynomial-like maps are proper holomorphic maps $f: U \rightarrow V$, where $U \Subset$ $V \subset \mathbb{C}^{k}$ and $V$ is convex. They must be thought of as a generalization of the endomorphisms of $\mathbb{P}^{k}$ (their lifts give rise to polynomial-like maps). The dynamical study of polynomial-like maps in arbitrary dimension was undertaken by Dinh-Sibony [12]. They proved that such systems admit a canonically defined measure of maximal entropy. Moreover, if we restrict to polynomial-like maps of large topological degree (those for which, roughly speaking, the topological degree dominates the other dynamical degrees, see Definition 2.7) this equilibrium measure enjoys much of the properties of its counterpart for endomorphisms of $\mathbb{P}^{k}$, but still does not admit any potential.

In order to state the main results of this paper we need to give some preliminary definitions. The first one, introduced in [5] for endomorphisms of $\mathbb{P}^{k}$, concerns Misi- 
urewicz parameters. These are the higher-dimensional analogue of the rational maps with a critical point non-persistently landing on a repelling cycle and are the key to understanding the interplay between bifurcation and critical dynamics.

Definition 1.1 Let $f_{\lambda}: U_{\lambda} \rightarrow V_{\lambda}$ be a holomorphic family of polynomial-like maps and let $C_{f}$ be the critical set of the map $f(\lambda, z)=\left(\lambda, f_{\lambda}(z)\right)$. A point $\lambda_{0}$ of the parameter space $M$ is called a Misiurewicz parameter if there exist a neighbourhood $N_{\lambda_{0}} \subset M$ of $\lambda_{0}$ and a holomorphic map $\sigma: N_{\lambda_{0}} \rightarrow \mathbb{C}^{k}$ such that:

1. for every $\lambda \in N_{\lambda_{0}}, \sigma(\lambda)$ is a repelling periodic point;

2. $\sigma\left(\lambda_{0}\right)$ is in the Julia set $J_{\lambda_{0}}$ of $f_{\lambda_{0}}$;

3. there exists an $n_{0}$ such that $\left(\lambda_{0}, \sigma\left(\lambda_{0}\right)\right)$ belongs to some component of $f^{n_{0}}\left(C_{f}\right)$;

4. $\sigma\left(N_{\lambda_{0}}\right)$ is not contained in a component of $f^{n_{0}}\left(C_{f}\right)$ satisfying 3 .

Here the Julia set is the support of the equilibrium measure, see Definition 2.4. Our main result is the following (see Definition 2.6 for the definition of the Lyapunov function $L$ ).

Theorem A Let $f_{\lambda}$ be a holomorphic family of polynomial-like maps of large topological degree. Assume that $\lambda_{0}$ is a Misiurewicz parameter. Then, $\lambda_{0} \in \operatorname{Supp} d d^{c} L$.

This statement in the case of $\mathbb{P}^{k}[5]$ relies on the generalization of De Marco's formula and the existence of a potential for the equilibrium measures. Here, we develop a different and more geometric approach. The next proposition is the technical counterpart of Theorem A. The dynamical degree $d_{k-1}^{*}$ of a polynomial-like map will be introduced in Definition 2.2. It is strictly smaller than $d_{t}$.

Proposition A' Let $f(\lambda, z)=\left(\lambda, f_{\lambda}(z)\right)$ be a holomorphic family of polynomial-like maps of large topological degree $d_{t}$. Let $\Lambda$ be an open and relatively compact subset of the parameter space. Let $d_{k-1}^{*}:=\sup _{\lambda \in \Lambda} d_{k-1}^{*}\left(f_{\lambda}\right)<d_{t}$. Then

$$
\begin{aligned}
\left\|d d^{c} L\right\|_{\Lambda} \neq 0 & \Leftrightarrow \limsup _{n \rightarrow \infty} \frac{1}{n} \log \left\|\left(f^{n}\right)_{*} C_{f}\right\|_{\Lambda \times \mathbb{C}^{k}}>\log d_{k-1}^{*} \\
& \Leftrightarrow \limsup _{n \rightarrow \infty} \frac{1}{n} \log \left\|\left(f^{n}\right)_{*} C_{f}\right\|_{\Lambda \times \mathbb{C}^{k}}=d_{t} .
\end{aligned}
$$

Moreover, if the family admits a Misiurewicz parameter then

$$
\limsup _{n \rightarrow \infty} \frac{1}{n} \log \left\|\left(f^{n}\right)_{*} C_{f}\right\|_{\Lambda \times \mathbb{C}^{k}}>\log d_{k-1}^{*} .
$$

Here $\left\|\left(f^{n}\right)_{*} \mathrm{C}_{f}\right\|_{\Lambda \times \mathbb{C}^{k}}$ denotes the mass (as a positive closed current in the product space $\Lambda \times \mathbb{C}^{k}$ ) of the integration current (with multiplicity) on the hypersurface $f^{n}\left(C_{f}\right)$, which is equivalent to its volume.

The proof of the first assertion is based on the theory of slicing of currents and more precisely on the use of equilibrium currents (see [14]), which was initiated by Pham [26]. To prove the second assertion, we exploit the mixing property of the equilibrium 
measure and construct a cylinder $T_{0}$ in the product space with more than $c^{n}$ smaller tubes $T_{n, i}$, contained in it, sent biholomorphically to $T_{0}$ by $f^{n}$. We can arrange the local picture in such a way that the component of the postcritical hypersurface $f^{n_{0}}\left(C_{f}\right)$ meeting the repelling cycle must cross all the $T_{n, i}$ 's. By applying $f^{n}$, the intersections between $f^{n_{0}}\left(C_{f}\right)$ and the small tubes $T_{n, i}$ are all sent to analytic subsets of $T_{0}$, whose volume is thus uniformly bounded from below.

Both statements in Proposition A' have an interest in their own. In particular, the first estimate can be seen as a generalization of the fact that, in dimension one, the bifurcation locus coincides with the non-normality locus of some critical orbit. The underlying geometry in the proof of the second statement may also lead to estimates for the Hausdorff dimension of the bifurcation loci (see [4]).

In the second part of the paper we exploit Theorem A to generalize the theory developed in [5] to the setting of polynomial-like maps of large topological degree and to arbitrary families of endomorphisms of $\mathbb{P}^{k}$. As we shall see, the main task left is to establish an analogous result in our setting of the equivalence between the holomorphic motion of the repelling cycles and the holomorphic motion of the Julia sets. To this aim, consider the set

$$
\mathcal{J}:=\left\{\gamma: M \rightarrow \mathbb{C}^{k}: \gamma \text { is holomorphic and } \gamma(\lambda) \in J_{\lambda} \text { for every } \lambda \in M\right\}
$$

The family $\left(f_{\lambda}\right)_{\lambda}$ naturally induces an action $\mathcal{F}$ on $\mathcal{J}$, by $(\mathcal{F} \cdot \gamma)(\lambda):=f_{\lambda}(\gamma(\lambda))$. We denote by $\Gamma_{\gamma}$ the graph of the element $\gamma \in \mathcal{J}$ in the product space. The following is the analogue of holomorphic motion of Julia sets in this setting (see [5]).

Definition 1.2 An equilibrium lamination is an $\mathcal{F}$-invariant subset $\mathcal{L}$ of $\mathcal{J}$ such that

1. $\Gamma_{\gamma} \cap \Gamma_{\gamma^{\prime}}=\varnothing$ for every distinct $\gamma, \gamma^{\prime} \in \mathcal{L}$;

2. $\mu_{\lambda}(\{\gamma(\lambda), \gamma \in \mathcal{L}\})=1$ for every $\lambda \in M$, where $\mu_{\lambda}$ is the equilibrium measure of $f_{\lambda}$;

3. $\Gamma_{\gamma}$ does not meet the grand orbit of the critical set of $f$ for every $\gamma \in \mathcal{L}$;

4. the map $\mathcal{F}: \mathcal{L} \rightarrow \mathcal{L}$ is $d^{k}$ to 1 .

We also introduce a weak notion of holomorphic motion for the repelling cycles in the Julia set, the repelling $J$-cycles. Recall that in higher dimensions repelling points may be outside the Julia set $([18,21])$.

Definition 1.3 Let $f_{\lambda}: U_{\lambda} \rightarrow V_{\lambda}$ be a holomorphic family of polynomial-like maps of large topological degree with parameter space $M$. We say that asymptotically all $J$-cycles move holomorphically on $M$ if there exists a subset $\mathcal{P}=\cup_{n} \mathcal{P}_{n} \subset \mathcal{J}$ such that

1. $\operatorname{Card} \mathcal{P}_{n}=d^{n}+o\left(d^{n}\right)$;

2. every $\gamma \in \mathcal{P}_{n}$ is $n$-periodic; and

3. for every $M^{\prime} \Subset M$, asymptotically every element of $\mathcal{P}$ is repelling, i.e.,

$$
\frac{\text { Card }\left\{\gamma \in \mathcal{P}_{n}: \gamma(\lambda) \text { is repelling for every } \lambda \in M^{\prime}\right\}}{\text { Card } \mathcal{P}_{n}} \rightarrow 1 .
$$

The following result, giving the desired equivalence, is established in Sect. 4.3. 
Theorem B Let $f_{\lambda}$ be any holomorphic family of polynomial-like maps of large topological degree $d_{t} \geq 2$, or of endomorphisms of $\mathbb{P}^{k}$. There exists an equilibrium lamination if and only if asymptotically all $J$-cycles move holomorphically.

The proof of an analogous statement (giving the holomorphic motion of all repelling $J$-cycles) on $\mathbb{P}^{k}([5])$ needs some assumptions on the family to avoid possible phenomena of non-linearizability. We explicitly remark that the proof that we present, although it gives a slightly weaker result, has the added value that can be applied to every family. It therefore provides a satisfactory extension of the theory of [5] to arbitrary holomorphic families of endomorphisms of $\mathbb{P}^{k}$. Our strategy is a generalization to the space of holomorphic graphs of a method due to Briend-Duval [8]. In their situation, they could recover the equidistribution of the repelling periodic points with respect to the equilibrum measure from the fact that all Lyapunov exponenents are strictly positive. We first generalize this idea to a self map of a compact metric space with good expansion properties, and then apply it in our setting.

In view of Theorems A and B we can characterize stability for polynomial-like maps of large topological degree as follows.

Theorem C Let $f_{\lambda}$ be a holomorphic family of polynomial-like maps of large topological degree $d_{t} \geq 2$. Assume that the parameter space is simply connected. Then the following are equivalent:

A.1 asymptotically all J-cycles move holomorphically;

A.2 there exists an equilibrium lamination for $f$;

A.3 the Lyapunov function is pluriharmonic;

A.4 there are no Misiurewicz parameters.

The implication A.3 $\Rightarrow$ A.4 is obtained from Theorem A and the implication A.2 $\Rightarrow$ A. 1 is given by Theorem B. For the other implications, the strategy is essentially the same as the one for $\mathbb{P}^{k}$; minor work is needed to adapt the proofs to the current setting. We shall thus just focus on the differences, referring the reader to [6] for the omitted details.

To conclude, let us mention two known differences with respect to the dimension one. Even for families of endomorphisms of $\mathbb{P}^{k}$, the conditions in Theorem $\mathrm{C}$ are in general not equivalent to the Hausdorff continuity of the Julia sets (see [7]). Moreover, these conditions do not define a dense subset of the parameter space (see $[7,15,29])$.

\section{Families of polynomial-like maps}

Unless otherwise stated, all the results presented here are due to Dinh-Sibony (see $[12,14])$.

\subsection{Polynomial-like maps}

The starting definition is the following.

Definition 2.1 A polynomial-like map is a proper holomorphic map $g: U \rightarrow V$, where $U \Subset V$ are open subsets of $\mathbb{C}^{k}$ and $V$ is convex. 
A polynomial-like map is in particular a (branched) holomorphic covering from $U$ to $V$, of a certain degree $d_{t}$ (the topological degree of $g$ ). We shall always assume that $d_{t} \geq 2$. The filled Julia set $K$ is the subset of $U$ given by $K:=\bigcap_{n \geq 0} g^{-n}(U)$. Notice that $g^{-1}(K)=K=g(K)$ and thus $(K, g)$ is a well-defined dynamical system. Lifts of endomorphisms of $\mathbb{P}^{k}$ are polynomial-like maps. Moreover, polynomial-like maps are stable under small perturbations of the map.

For a polynomial-like map $g$, the knowledge of the topological degree is not enough to predict the volume growth of analytic subsets. We are thus lead to consider more general degrees than the topological one. In the following definition, we denote by $\omega$ the standard Kähler form on $\mathbb{C}^{k}$. Moreover, recall that the mass of a positive $(p, p)$ current $T$ on a Borel set $X$ is given by $\|T\|_{X}=\int_{X} T \wedge \omega^{k-p}$.

Definition 2.2 Given a polynomial-like map $g: U \rightarrow V$, the $*$-dynamical degree of order $p$, for $0 \leq p \leq k$, of $g$ is given by

$$
d_{p}^{*}(g):=\limsup _{n \rightarrow \infty} \sup _{S}\left\|\left(g^{n}\right)_{*}(S)\right\|_{W}^{1 / n}
$$

where $W \Subset V$ is a neighbourhood of $K$ and the sup is taken over all positive closed $(k-p, k-p)$-currents of mass less than or equal to 1 on a fixed neighbourhood $W^{\prime} \Subset V$ of $K$.

It is quite straighforward to check that this definition does not depend on the particular neighbourhoods $W$ and $W^{\prime}$ chosen for the computations. Moreover, the following hold: $d_{0}^{*}=1, d_{k}^{*}=d_{t}, d_{p}^{*}\left(g^{m}\right)=\left(d_{p}^{*}(g)\right)^{m}$ and a relation $d_{p}^{*}<d_{t}$ is preserved by small perturbations.

Theorem 2.3 Let $g: U \rightarrow V$ be a polynomial-like map and $v$ be a probability measure supported on $V$ which is defined by an $L^{1}$ form. Then $d_{t}^{-n}\left(g^{n}\right)^{*} v$ converge to a probability measure $\mu$ which does not depend on $v$. Moreover, for any psh function $\phi$ on a neighbourhood of $K$ the sequence $d_{t}^{-n}\left(g^{n}\right)_{*} \phi$ converge to $\langle\mu, \phi\rangle \in\{-\infty\} \cup \mathbb{R}$. The measure $\mu$ is ergodic, mixing and satisfies $g^{*} \mu=d_{t} \mu$.

The convergence of $d_{t}^{-n}\left(g^{n}\right)_{*} \phi$ in Theorem 2.3 is in $L_{l o c}^{p}$ for every $1 \leq p<\infty$ if $\langle\mu, \phi\rangle$ is finite. Otherwise, if $\langle\mu, \phi\rangle=-\infty$ the convergence is uniform on compact subsets.

Definition 2.4 The measure $\mu$ given by Theorem 2.3 is called the equilibrium measure of $g$. The support of $\mu$ is the Julia set of $g$, denoted with $J_{g}$.

The assumption on $v$ to be defined by an $L^{1}$ form can be relaxed to just asking that $v$ does not charge pluripolar sets. The following Theorem ensures that $\mu$ itself does not charge the critical set of $g$. Notice that $\mu$ may charge proper analytic subsets. This is a difference with respect to the case of endomorphisms of $\mathbb{P}^{k}$.

Theorem 2.5 Let $f: U \rightarrow V$ be a polynomial-like map of degree $d_{t}$. Then $\left\langle\mu, \log \left|\mathrm{Jac}_{g}\right|\right\rangle \geq \frac{1}{2} \log d_{t}$. 
A consequence of Theorem 2.5 (by Parry Theorem [25]) is that the equilibrium measure has entropy at least $\log d_{t}$. It is thus a measure of maximal entropy (see [14]). Another important consequence is the existence, by Oseledets Theorem [24] of the Lyapunov exponents $\chi_{i}(g)$ of a polynomial-like map with respect to the equilibrium measure $\mu$.

Definition 2.6 The Lyapunov function $L(g)$ is the sum

$$
L(g)=\sum \chi_{i}(g)
$$

By Oseledets and Birkhoff Theorems, it follows that $L(g)=\langle\mu, \log |\mathrm{Jac}|\rangle$. By Theorem 2.5, we thus have $L(g) \geq \frac{1}{2} \log d_{t}$ for every polynomial-like map $g$.

\subsection{Maps of large topological degree}

Recall that the $*$-dynamical degrees were defined in Definition 2.2.

Definition 2.7 A polynomial-like map is of large topological degree if $d_{k-1}^{*}<d_{t}$.

Notice that holomorphic endomorphisms of $\mathbb{P}^{k}$ (and thus their polynomial-like lifts) satify the above estimate. Morever, a small perturbation of a polynomial-like map of large topological degree still satisfy this property.

The equilibrium measure of a polynomial-like map of large topological degree integrates psh functions, and thus in particular does not charge pluripolar sets (see [14, Theorem 2.33]).

We end this section recalling two equidistribution properties $[12,14]$ of the equilibrium measure of a polynomial-like map of large topological degree.

Theorem 2.8 Let $g: U \rightarrow V$ be a polynomial-like map of large topological degree $d_{t} \geq 2$.

1. Let $R_{n}$ denote the set of repelling n-periodic points in the Julia set $J$. Then

$$
\frac{1}{d_{t}^{n}} \sum_{a \in R_{n}} \delta_{a} \rightarrow \mu
$$

2. There exists a proper analytic set $\mathscr{E}$ (possibly empty) contained in the postcritical set of $g$ such that

$$
d_{t}^{-n}\left(g^{n}\right)^{*} \delta_{a}=\frac{1}{d_{t}^{n}} \sum_{g^{n}(b)=a} \delta_{b} \rightarrow \mu
$$

if and only if a does not belong to the orbit of $\mathscr{E}$.

An important consequence of the proof of (the second part of) Theorem 2.8 is that all Lyapunov exponents of a polynomial-like map of large topological degree are 
bounded below by $\frac{1}{2} \log \frac{d_{t}}{d_{k-1}^{*}}>0$. This property will play a very important role in the proof of Theorem A. It is also crucial to establish the existence of an equilibrium lamination from the motion of the repelling points, see $[5,6]$.

\subsection{Holomorphic families}

We now come to the main object of our study.

Definition 2.9 Let $M$ be a complex manifold and $\mathcal{U} \Subset \mathcal{V}$ be connected open subsets of $M \times \mathbb{C}^{k}$. Denote by $\pi_{M}$ the standard projection $\pi_{M}: M \times \mathbb{C}^{k} \rightarrow M$. Suppose that for every $\lambda \in M$, the two sets $U_{\lambda}:=\mathcal{U} \cap \pi^{-1}(\lambda)$ and $V_{\lambda}:=\mathcal{V} \cap \pi^{-1}(\lambda)$ satisfy $\emptyset \neq U_{\lambda} \Subset V_{\lambda} \Subset \mathbb{C}^{k}$, that $U_{\lambda}$ is connected and that $V_{\lambda}$ is convex. Moreover, assume that $U_{\lambda}$ and $V_{\lambda}$ depend continuously on $\lambda$ (in the sense of Hausdorff). A holomorphic family of polynomial-like maps is a proper holomorphic map $f: \mathcal{U} \rightarrow \mathcal{V}$ fibered over $M$, i.e., of the form

$$
\begin{aligned}
f: \mathcal{U} & \rightarrow \mathcal{V} \\
(\lambda, z) & \mapsto\left(\lambda, f_{\lambda}(z)\right) .
\end{aligned}
$$

From the definition, $f$ has a well defined topological degree, that we shall always denote with $d_{t}$ and assume to be greater than 1 . In particular, each $f_{\lambda}: U_{\lambda} \rightarrow V_{\lambda}$ is a polynomial-like map, of degree $d_{t}$. We shall denote by $\mu_{\lambda}, J_{\lambda}$ and $K_{\lambda}$ the equilibrium measure, the Julia set and the filled Julia set of $f_{\lambda}$, while $C_{f}, \mathrm{Jac}_{f}$ and $\mathrm{C}_{f}$ will be the critical set, the determinant of the (complex) jacobian matrix of $f$ and the integration current $d d^{c} \log \left|\operatorname{Jac}_{f}\right|$. We may drop the subscript $f$ if no confusion arises.

It is immediate to see that the filled Julia set $K_{\lambda}$ varies upper semicontinuously with $\lambda$ for the Hausdoff topology. This allows us, when dealing with local problems to assume that $V_{\lambda}$ does not depend on $\lambda$, i.e., that $\mathcal{V}=M \times V$, with $V$ an open, convex and relatively compact subset of $\mathbb{C}^{k}$. On the other hand, the Julia set is lower semicontinuous in $\lambda$ for a family of maps of large topological degree ([14]).

We now recall the construction, due to Pham [26], of an equilibrium current for a family of polynomial-like maps. This is based on the following Theorem. We recall that a horizontal current on a product space $M \times V$ is a current whose support is contained in $M \times L$, where $L$ is some compact subset of $V$. We refer to [17] (see also $[14,20,28])$ for the basics on slicing.

Theorem 2.10 (Dinh-Sibony [13], Pham [26]) Let $M$ and $V$ two complex manifolds, of dimension $m$ and $k$. Let $\mathcal{R}$ be a horizontal positive closed $(k, k)$-current and $\psi$ a psh function on $M \times V$. Then

1. the slice $\langle\mathcal{R}, \pi, \lambda\rangle$ of $\mathcal{R}$ at $\lambda$ with respect to the projection $\pi: M \times V \rightarrow M$ exists for every $\lambda \in M$, and its mass is independent from $\lambda$;

2. the function $g_{\psi, \mathcal{R}}(\lambda):=\langle\mathcal{R}, \pi, \lambda\rangle(\psi(\lambda, \cdot))$ is psh or identically $-\infty$.

If $\left\langle\mathcal{R}, \pi, \lambda_{0}\right\rangle\left(\psi\left(\lambda_{0}, \cdot\right)\right)>-\infty$ for some $\lambda_{0} \in M$, then

3. the product $\psi \mathcal{R}$ is well defined; 
4. for every $\Omega$ continuous form of maximal degree compactly supported on $M$ we have

$$
\int_{M}\langle\mathcal{R}, \pi, \lambda\rangle(\psi) \Omega(\lambda)=\left\langle\mathcal{R} \wedge \pi^{*}(\Omega), \psi\right\rangle .
$$

In particular, the pushforward $\pi_{*}(\psi \mathcal{R})$ is well defined and coincides with the psh function $g_{\psi, \mathcal{R}}$.

Consider now a family of polynomial-like maps $f: \mathcal{U} \rightarrow \mathcal{V}=M \times V$. Let $\theta$ be a smooth probability measure compactly supported in $V$ and consider the (positive and closed) smooth $(k, k)$-currents on $M \times V$ defined by induction as

$$
\left\{\begin{array}{l}
S_{0}=\pi_{V}^{*}(\theta) \\
S_{n}:=\frac{1}{d_{t}} f^{*} S_{n-1}=\frac{1}{d_{t}^{n}}\left(f^{n}\right)^{*} S_{0} .
\end{array}\right.
$$

The $S_{n}$ ' are in particular horizontal positive closed $(k, k)$-currents on $M \times V$, whose slice mass is equal to 1 . Moreover, since by definition we have $\left\langle S_{0}, \pi, \lambda\right\rangle=\theta$ for every $\lambda \in M$, we have that $\left\langle S_{n}, \pi, \lambda\right\rangle=\frac{1}{d_{t}^{n}}\left(f_{\lambda}^{n}\right)^{*} \theta$. In particular, since every $f_{\lambda}: U_{\lambda} \rightarrow V$ is a polynomial-like map, for every $\lambda \in M$ we have $\left\langle S_{n}, \pi, \lambda\right\rangle \rightarrow \mu_{\lambda}$. The following Theorem ensures that the limits of the sequence $S_{n}$ have slices equal to $\mu_{\lambda}$.

Theorem 2.11 (Pham) Let $f: \mathcal{U} \rightarrow \mathcal{V}$ be a holomorphic family of polynomial-like maps. Up to a subsequence, the forms $S_{n}$ defined by (2.2) converge to a positive closed $(k, k)$-current $\mathcal{E}$ on $\mathcal{V}$, supported on $\cup_{\lambda}\{\lambda\} \times K_{\lambda}$, such that for every $\lambda \in M$ the slice $\langle\mathcal{E}, \pi, \lambda\rangle$ exists and is equal to $\mu_{\lambda}$.

Definition 2.12 An equilibrium current for $f$ is a positive closed current $\mathcal{E}$ on $\mathcal{V}$, supported on $\cup_{\lambda}\{\lambda\} \times K_{\lambda}$, such that $\langle\mathcal{E}, \pi, \lambda\rangle=\mu_{\lambda}$ for every $\lambda \in M$.

Given an equilibrium current $\mathcal{E}$ for $f$, the product $\log |\mathrm{Jac}| \cdot \mathcal{E}$ (and so also the intersection $\left.\mathcal{E} \wedge \mathrm{C}_{f}=d d^{c}(\log |\mathrm{Jac}| \cdot \mathcal{E})\right)$ is thus well defined (by Theorems 2.10 and 2.5). Moreover, the distribution $\pi_{*}(\log |\mathrm{Jac}| \cdot \mathcal{E})$ is represented by the (plurisubharmonic) function $\lambda \mapsto\left\langle\mu_{\lambda}, \log |\operatorname{Jac}(\lambda, \cdot)|\right\rangle$. Notice that, while the product $\log |\mathrm{Jac}| \cdot \mathcal{E}$ a priori depends on the particular equilibrium current $\mathcal{E}$, the pushforward by $\pi$ is independent from the particular choice (by (2.1)). By Oseledets and Birkhoff theorems the function $\lambda \mapsto\left\langle\mu_{\lambda}, \log |\operatorname{Jac}(\lambda, \cdot)|\right\rangle$ coincides with the Lyapunov function $L(\lambda)$, i.e., the sum of the Lyapunov exponents of $f_{\lambda}$ with respect to $\mu_{\lambda}$ (see Definition 2.6). The following definition is then well posed.

Definition 2.13 Let $f: \mathcal{U} \rightarrow \mathcal{V}$ be a holomorphic family of polynomial-like maps. The bifurcation current of $f$ is the positive closed $(1,1)$-current on $M$ given by

$$
T_{b i f}:=d d^{c} L(\lambda)=\pi_{*}\left(\mathrm{C}_{f} \wedge \mathcal{E}\right)
$$

where $\mathcal{E}$ is any equilibrium current for $f$. 
We conclude with two results concerning the approximation of the current $u \mathcal{E}$ for $u$ psh, that we shall need in Sect. 3. The first is due to Dinh and Sibony and is a crucial step in the proof of the Holder continuity of the Lyapunov function (see [14, Theorem 2.34] for the result at a fixed parameter $\lambda$, and [14, Theorem 2.49] for the uniformity in $\lambda$ ).

Lemma 2.14 (Dinh-Sibony) Let $f: \mathcal{U} \rightarrow \mathcal{V}=M \times V$ be a holomorphic family of polynomial-like maps of large topological degree. Let $\theta$ be a smooth positive measure compactly supported on $V$ and $u$ be a psh function on $M \times V$ such that the family of the restrictions $u(\lambda, \cdot)$ is bounded on some common neighbourhood of the filled Julia sets $K_{\lambda}$. Then, for every $\lambda_{0} \in M$ there exist a neighbourhood $\lambda_{0} \in M_{0} \Subset M$ and $a$ constant $\alpha<1$ such that

$$
\left\|\left\langle d_{t}^{-n}\left(f_{\lambda}^{n}\right)^{*} \theta, u(\lambda, \cdot)\right\rangle-\left\langle\mu_{\lambda}, u(\lambda, \cdot)\right\rangle\right\|_{M_{0}, \infty}<\alpha^{n}
$$

Lemma 2.15 Let $f: \mathcal{U} \rightarrow \mathcal{V}=M \times V$ be a holomorphic family of polynomial-like maps, with $\operatorname{dim} M=m$. Let $\mathcal{E}$ be an equilibrium current and $S_{n}$ be a sequence of smooth forms as in (2.2). Then for every smooth $(m-1, m-1)$-form $\Omega$ compactly supported on $M$ we have

$$
\left\langle C_{f} \wedge S_{n}, \pi^{*}(\Omega)\right\rangle \rightarrow\left\langle C_{f} \wedge \mathcal{E}, \pi^{*}(\Omega)\right\rangle
$$

Notice that the convergence holds without the need of taking the subsequence (recall that the right hand side is independent from the subsequence used to compute $\mathcal{E}$ ). Moreover, we do not need to restrict $M$ to get the statement since $\Omega$ is compactly supported. This also follows from the compactness of horizontal positive closed currents with bounded slice mass, see [13].

Proof It is enough to prove that, for every $u$ psh such that there exists $\lambda_{0} \in M$ such that $\left\langle\mu_{\lambda_{0}}, u\left(\lambda_{0}, \cdot\right)\right\rangle>-\infty$, for every continuous form $\Omega$ of maximal degree and compactly supported on $M$, we have

$$
\left\langle u S_{n}, \pi^{*}(\Omega)\right\rangle \rightarrow\left\langle u \mathcal{E}, \pi^{*}(\Omega)\right\rangle
$$

where the right hand side is well defined by Theorem 2.10 . Notice that the assumption $\left\langle\mu_{\lambda_{0}}, u\left(\lambda_{0}, \cdot\right)\right\rangle>-\infty$ at some $\lambda_{0}$ is automatic if the family is of large topological degree, see [14, Theorem 2.33], and is always satisfied when $u(\lambda, \cdot)=\log |\operatorname{Jac}(\lambda, \cdot)|$ (which is the function needed for the statement).

We can suppose that $\Omega$ is a positive volume form, since we can decompose it in its positive and negative parts $\Omega=\Omega^{+}-\Omega^{-}$and prove the statement for $\Omega^{+}$and $\Omega^{-}$separately. Moreover, by means of a partition of unity on $M$, we can also assume that $\mathcal{E}$ is horizontal. By Theorem 2.10, the product $u \mathcal{E}$ is well defined and the identity (2.1) holds with both $\mathcal{R}=\mathcal{E}$ or $S_{n}$ and $\psi=u$. So, it suffices to check that

$$
\int_{M}\left\langle S_{n}, \pi, \lambda\right\rangle(u) \Omega(\lambda) \rightarrow \int_{M}\langle\mathcal{E}, \pi, \lambda\rangle(u) \Omega(\lambda) .
$$


The assertion then follows since the slices of $\mathcal{E}$, and thus also the right hand side, are independent from the particular equilibrium current chosen.

Set $\phi_{n}(\lambda):=\left\langle S_{n}, \pi, \lambda\right\rangle(u(\lambda, \cdot))$ and $\phi(\lambda):=\langle\mathcal{E}, \pi, \lambda\rangle(u(\lambda, \cdot))=\left\langle\mu_{\lambda}, u(\lambda, \cdot)\right\rangle$. By Theorem 2.10, the $\phi_{n}$ 's and $\phi$ are psh functions on $M$. Moreover, at $\lambda$ fixed, we have (recalling the definition (2.2) of the $S_{n}$ 's and the fact that $d_{t}^{-n}\left(f_{\lambda}^{n}\right)_{*} u(\lambda, \cdot) \rightarrow$ $\left\langle\mu_{\lambda}, u(\lambda, \cdot)\right\rangle$ since $u(\lambda, \cdot)$ is psh, see Theorem 2.3)

$$
\begin{aligned}
\phi_{n}(\lambda) & =\left\langle S_{n}, \pi, \lambda\right\rangle u(\lambda, \cdot)=\left\langle\frac{1}{d_{t}^{n}}\left(f_{\lambda}^{n}\right)^{*}(\theta), u(\lambda, \cdot)\right\rangle=\left\langle\theta, \frac{1}{d_{t}^{n}}\left(f_{\lambda}^{n}\right)_{*} u(\lambda, \cdot)\right\rangle \\
& \rightarrow\left\langle\theta,\left\langle\mu_{\lambda}, u(\lambda, \cdot)\right\rangle\right\rangle=\left\langle\mu_{\lambda}, u(\lambda, \cdot)\right\rangle=\phi(\lambda) .
\end{aligned}
$$

Since $u$ is upper semicontinuous (and thus locally bounded) all the $\phi_{n}$ 's are bounded from above. This, together with the fact that they converge pointwise to $\phi$, gives that the convergence $\phi_{n} \rightarrow \phi$ happens in $L_{l o c}^{1}$, and the assertion is proved.

\section{Misiurewicz parameters belong to $\operatorname{Supp} d d^{c} L$}

In this section we prove Proposition A' (and Theorem A). The idea will be to relate the mass of $d d^{c} L$ on a given open set $\Lambda$ of the parameter space with the growth of the mass of the currents $f_{*}^{n}[C]$ on the vertical set $\mathcal{V} \cap \pi_{M}^{-1}(\Lambda)$. Then, we will show how the presence of a Misiurewicz parameter allows us to get the desired estimate for the growth of the critical volume, permitting to conclude. We shall need the following lemma, whose proof is a simple adaptation of the one of [14, Proposition 2.7].

Lemma 3.1 Let $f: \mathcal{U} \rightarrow \mathcal{V}$ be a holomorphic family of polynomial-like maps. Let $\delta>d_{p}^{*}\left(f_{\lambda_{0}}\right)$. There exists a constant $C$ such that, for $\lambda$ sufficiently close to $\lambda_{0}$, we have $\left\|\left(f_{\lambda}^{n}\right)_{*}(S)\right\|_{U_{\lambda}} \leq C \delta^{n}$ for every $n \in \mathbb{N}$ and every closed positive $(k-p, k-p)$-current $S$ of mass less than or equal to 1 on $U_{\lambda}$.

The following Theorem (which implies the first part of Proposition A') gives the relation between the mass of $d d^{c} L$ and the growth of the mass of $\left(f^{n}\right)_{*} \mathrm{C}_{f}$. We recall that $\mathrm{C}_{f}=d d^{c} \log \left|\mathrm{Jac}_{f}\right|$ is the integration on the critical set of $f$, counting the multiplicity. We set $\mathcal{U}_{\Lambda}:=\mathcal{U} \cap\left(\Lambda \times \mathbb{C}^{k}\right)$ and $\mathcal{V}_{\Lambda}:=\mathcal{V} \cap\left(\Lambda \times \mathbb{C}^{k}\right)$

Theorem 3.2 Let $f: \mathcal{U} \rightarrow \mathcal{V}$ be a holomorphic family of polynomial-like maps. Set $d_{k-1}^{*}:=\sup _{\lambda \in M} d_{k-1}^{*}\left(f_{\lambda}\right)$, and assume that $d_{k-1}^{*}$ is finite. Let $\delta$ be greater than $d_{k-1}^{*}$. Then for any open subset $\Lambda \Subset M$ there exist positive constants $c_{1}^{\prime}, c_{1}$ and $c_{2}$ such that, for every $n \in \mathbb{N}$,

$$
c_{1}^{\prime}\left\|d d^{c} L\right\|_{\Lambda} d_{t}^{n} \leq\left\|\left(f^{n}\right)_{*} C_{f}\right\|_{\mathcal{U}_{\Lambda}} \leq c_{1}\left\|d d^{c} L\right\|_{\Lambda} d_{t}^{n}+c_{2} \delta^{n} .
$$

In particular, if

$$
\limsup _{n \rightarrow \infty} \frac{1}{n} \log \left\|\left(f^{n}\right)_{*} C_{f}\right\|_{\mathcal{U}_{\Lambda}}>\log d_{k-1}^{*},
$$

then $\Lambda$ intersects the bifurcation locus. 
Notice that $\left(f^{n}\right)_{*} \mathrm{C}_{f}$ actually denotes the current on $\mathcal{U}_{\Lambda}$ which is the pushforward by the proper map $f^{n}: f^{-n}\left(\mathcal{U}_{\Lambda}\right) \rightarrow \mathcal{U}_{\Lambda}$ of the current $\mathbf{C}_{f}$ on $f^{-n}\left(\mathcal{U}_{\Lambda}\right)$.

Proof The problem is local. We can thus assume that $\mathcal{V}=M \times V$, where $V$ is convex. Moreover, there exists some open convex set $\tilde{U}$ such that $U_{\lambda} \Subset \tilde{U} \Subset V$ for every $\lambda \in M$.

Let us denote by $\omega_{V}$ and $\omega_{M}$ the standard Kähler forms on $\mathbb{C}^{k}$ and $\mathbb{C}^{m}$. By abuse of notation, we denote by $\omega_{V}+\omega_{M}$ the Kähler form $\pi_{V}^{*} \omega_{V}+\pi_{M}^{*} \omega_{M}$ on the product space $M \times V$. Since both $\omega_{V}^{k+1}$ and $\omega_{M}^{m+1}$ are zero, by the definition of mass we have

$$
\begin{aligned}
\left\|\left(f^{n}\right)_{*} \mathbf{C}_{f}\right\|_{\mathcal{U}_{\Lambda}}= & \int_{\mathcal{U}_{\Lambda}}\left(f^{n}\right)_{*} \mathbf{C}_{f} \wedge\left(\omega_{V}+\omega_{M}\right)^{k+m-1} \\
= & \left(\begin{array}{c}
k+m-1 \\
k
\end{array}\right) \int_{\mathcal{U}_{\Lambda}}\left(f^{n}\right)_{*} \mathbf{C}_{f} \wedge \omega_{V}^{k} \wedge \omega_{M}^{m-1} \\
& +\left(\begin{array}{c}
k+m-1 \\
k-1
\end{array}\right) \int_{\mathcal{U}_{\Lambda}}\left(f^{n}\right)_{*} \mathrm{C}_{f} \wedge \omega_{V}^{k-1} \wedge \omega_{M}^{m}
\end{aligned}
$$

We shall bound the two integrals from above by means of $\left\|d d^{c} L\right\|_{\Lambda} d_{t}^{n}+\delta^{n}$ and $\delta^{n}$, respectively, and from below with $\left\|d d^{c} L\right\|_{\Lambda} d_{t}^{n}$ and 0 . Let us start with the first one. Let $\rho$ be a positive smooth function, compactly supported on $V$, equal to a constant $c_{\rho}$ on $\tilde{U}$ and such that the integral of $\rho$ is equal to 1 . Notice in particular that $\rho / c_{\rho}$ is equal to 1 on $\tilde{U}$ and has total mass $1 / c_{\rho}$. Then

$$
\int_{\mathcal{U}_{\Lambda}}\left(f^{n}\right)_{*} \mathrm{C}_{f} \wedge \omega_{V}^{k} \wedge \omega_{M}^{m-1} \leq \frac{1}{c_{\rho}} \int_{\mathcal{V}_{\Lambda}}\left(f^{n}\right)_{*} \mathbf{C}_{f} \wedge\left(\pi_{V}^{*} \rho \cdot \omega_{V}^{k}\right) \wedge \omega_{M}^{m-1}
$$

Consider the smooth $(k, k)$-forms $S_{n}:=\frac{\left(f^{n}\right)^{*}}{d_{t}^{n}}\left(\pi_{V}^{*} \rho \cdot \omega_{V}^{k}\right)$. By Theorem 2.11, every subsequence of $\left(S_{n}\right)_{n}$ has a further subsequence converging to an equilibrium current $\mathcal{E}^{\prime}$ (possibly depending on the subsequence). Let $S_{n_{i}}$ be such a subsequence. By Definition 2.13, we have $d d^{c} L=\pi_{*}\left(\mathrm{C}_{f} \wedge \mathcal{E}^{\prime}\right)$ for any possible limit $\mathcal{E}^{\prime}$. Since $f^{*} \omega_{M}=\omega_{M}$, we then have

$$
\begin{aligned}
d_{t}^{-n_{i}} \int_{\mathcal{V}_{\Lambda}}\left(f^{n_{i}}\right)_{*} \mathrm{C}_{f} & \wedge\left(\pi_{V}^{*} \rho \cdot \omega_{V}^{k}\right) \wedge \omega_{M}^{m-1}=\int_{f^{-n_{i}}\left(\mathcal{V}_{\Lambda}\right)} \mathrm{C}_{f} \wedge S_{n_{i}} \wedge \omega_{M}^{m-1} \\
& \rightarrow \int_{\mathcal{V}_{\Lambda}} \mathrm{C}_{f} \wedge \mathcal{E}^{\prime} \wedge \omega_{M}^{m-1}=\left\|d d^{c} L\right\|_{\Lambda}
\end{aligned}
$$

where the convergence follows from Lemma 2.15 (by means of a partition of unity on $\Lambda$ ). Since the limit is independent from the subsequence, the convergence above happens without the need of extraction In particular, if $\left\|d d^{c} L\right\| \neq 0$ we have

$$
\int_{\mathcal{V}_{\Lambda}}\left(f^{n}\right)_{*} \mathbf{C}_{f} \wedge\left(\pi_{V}^{*} \rho \cdot \omega_{V}^{k}\right) \wedge \omega_{M}^{m-1} \leq \tilde{c}_{1}\left\|d d^{c} L\right\|_{\Lambda} d_{t}^{n}
$$


for some positive constant $\tilde{c}_{1}$ and the desired bound from above follows. The bound from below is completely analogous, by means of a function $\rho$ equal to 1 on a neighbourhood of $\cup_{\lambda}\{\lambda\} \times K_{\lambda}$. To conclude the estimate of this term we just need to prove that if $\left\|d d^{c} L\right\|=0$ then the integral above is bounded by (a constant times) $\delta^{n}$ for some $\delta<d_{t}$. This is a consequence of Lemma 2.14. Indeed, setting $L_{n}(\lambda):=\int \log \left|\operatorname{Jac}_{f_{\lambda}}\right|\left(f_{\lambda}^{n}\right)^{*}\left(\rho \cdot \omega_{V}^{k}\right)$ and recalling that $L(\lambda)=\int \log \left|\operatorname{Jac}_{f_{\lambda}}\right| \mu_{\lambda}$, it follows from Lemma 2.14 that $\left|d_{t}^{-n} L_{n}(\lambda)-L(\lambda)\right|_{\infty}<\alpha^{n}$ for $n \gg 1$ and some $\alpha<1$. So,

$$
\begin{aligned}
d_{t}^{-n} \int_{\mathcal{V}_{\Lambda}}\left(f^{n}\right)_{*} \mathrm{C}_{f} \wedge\left(\pi_{V}^{*} \rho \cdot \omega_{V}^{k}\right) \wedge \omega_{M}^{m-1} & =\left\|d d^{c} d_{t}^{-n} L_{n}\right\|=\left\|d d^{c}\left(d_{t}^{-n} L_{n}-L\right)\right\| \\
& =\int d d^{c}\left(d_{t}^{-n} L_{n}-L\right) \wedge \omega_{M}^{m-1}
\end{aligned}
$$

and the estimate follows by using a cut off function and an integration by parts.

Let us then estimate the second integral. We claim that

$$
\int_{\Lambda \times \tilde{U}}\left(f^{n}\right)_{*} \mathrm{C}_{f} \wedge \omega_{V}^{k-1} \wedge \omega_{M}^{m}=\int_{\Lambda}\left\|\left(f_{\lambda}^{n}\right)_{*} \mathrm{C}_{f_{\lambda}}\right\|_{\tilde{U}} \omega_{M}^{m}
$$

where $\mathrm{C}_{f_{\lambda}}$ is the integration current (with multiplicity) on the critical set of $f_{\lambda}$. The assertion then follows since, by Lemma 3.1, the right hand side in (3.1) is bounded by $\tilde{c}_{2} \delta^{n}$, for some positive $\tilde{c}_{2}$.

Let us thus prove (3.1). By [28, p. 124] and [17, Theorem 4.3.2(7)], the slice $\left\langle\left(f^{n}\right)_{*} \mathbf{C}_{f}, \pi, \lambda\right\rangle$ of $\left(f^{n}\right)_{*} \mathbf{C}_{f}$ exists for almost every $\lambda \in \Lambda$ and is given by

$$
\left\langle\left(f^{n}\right)_{*} \mathbf{C}_{f}, \pi, \lambda\right\rangle=\left(f_{\lambda}^{n}\right)_{*}\left\langle\mathbf{C}_{f}, \pi, \lambda\right\rangle=\left(f_{\lambda}^{n}\right)_{*} \mathbf{C}_{f_{\lambda}} .
$$

Since $\omega_{V}^{k-1}$ is smooth, this implies that the slice of $\left(f^{n}\right)_{*} \mathbf{C}_{f} \wedge \omega_{V}^{k-1}$ exists for almost every $\lambda$ and is equal to the measure $\left(\left(f_{\lambda}^{n}\right)_{*} \mathrm{C}_{f_{\lambda}}\right) \wedge \omega_{V}^{k-1}$. The claim then follows from [17, Theorem 4.3.2] by integrating a partition of unity.

Now we aim to bound from below a subsequence of $\left(\left\|\left(f^{n}\right)_{*} \mathbf{C}_{f}\right\|_{\mathcal{U}_{\Lambda}}\right)_{n}$ in presence of a Misiurewicz parameter. The main tool to achieve this goal is given by the next proposition.

Proposition 3.3 Let $f: \mathcal{U} \rightarrow \mathcal{V}=\mathbb{D} \times V$ be a holomorphic family of polynomial-like maps of large topological degree $d_{t}$. Fix a ball $B \Subset V$ such that $B \cap J\left(f_{0}\right) \neq \emptyset$ and let $\delta$ be such that $0<\delta<d_{t}$. There exists a ball $A_{0} \subset B$, an $N>0$ and an $\eta>0$ such that $f^{N}$ admits at least $\delta^{N}$ inverse branches defined on the cylinder $\mathbb{D}_{\eta} \times A_{0}$, with image contained in $\mathbb{D}_{\eta} \times A_{0}$.

In the proof of the above proposition we shall first need to construct a ball $A \subset$ $B$ with the required number of inverse branches for $f_{0}$. This is done by means of the following general lemma. Fix any polynomial-like map $g: U \rightarrow V$ of large topological degree. Given any $A \subset V, n \in \mathbb{N}$ and $\rho>0$, denote by $C_{n}(A, \rho)$ the set 


$$
C_{n}(A, \rho):=\left\{h \mid \begin{array}{c}
h \text { is an inverse branch of } g^{n} \text { defined on } \bar{A} \\
\text { and such that } h(\bar{A}) \subset A \text { and } \operatorname{Lip} h_{\mid \bar{A}} \leq \rho
\end{array}\right\} .
$$

The following result, which is just a local version of [5, Proposition 3.8], is essentially due to Briend-Duval (see [8]).

Lemma 3.4 Let $g$ be a polynomial-like map of large topological degree $d_{t}$. Let $B$ be a ball intersecting $J$ and $\rho$ a positive number. There exists a ball A contained in $B$ and an $\alpha>0$ such that $\# C_{n}(A, \rho) \geq \alpha d_{t}^{n}$, for every $n$ sufficently large.

Proof (Proof of Proposition 3.3) Let $A \subset B$ be a ball given by an application of Lemma 3.4 to the map $f_{0}$, with $\rho=1 / 4$. There thus exists an $\alpha$ such that, for every sufficiently large $n$, the set $C_{n}(A, 1 / 4)$ defined as in (3.2) has at least $\alpha d_{t}^{n}$ elements. Fix $N$ sufficiently large such that $\delta^{N}<\alpha d_{t}^{N}$. Denote by $h_{i}$ the elements of $C_{N}(A, 1 / 4)$ and by $A_{i}$ the images $A_{i}:=h_{i}(A) \subset A$. By definition of inverse branches, the $A_{i}$ 's are all disjoint and $f_{0}^{N}$ induces a biholomorphism from every $A_{i}$ to $A$.

Take as $A_{0}$ any open ball relatively compact in $A$ and such that $\cup_{i} \bar{A}_{i} \Subset A_{0}$. Such an $A_{0}$ exists since $\cup_{i} \bar{A}_{i} \Subset A$. In particular, on $A_{0}$ the $h_{i}$ 's are well defined, with images (compactly) contained in the $A_{i}$ 's. By continuity, for $\eta$ small enough we have that these inverse branches for $f_{0}^{N}$ extend to inverse branches for $f^{N}$ on $\mathbb{D}_{\eta} \times A_{0}$, with images contained in $\mathbb{D}_{\eta} \times A_{0}$ (see [6] for details). This concludes the proof.

We can now prove the second part of Proposition A'.

Proof (Proof of Proposition A', second part) We prove that the existence of a Misiurewicz parameter implies that the mass of $\left(f^{n}\right)_{*} \mathrm{C}_{f}$ is asymptotically larger than $\tilde{d}^{n}$ (up to considering a subsequence), for some $\tilde{d}>d_{k-1}^{*}$.

Before starting proving the assertion, we make a few simplifications to the problem. Let $\sigma(\lambda)$ denote the repelling periodic point intersecting (but not being contained in) some component of $f^{n_{0}}(C)$ at $\lambda=\lambda_{0}$ and such that $\sigma(0) \in J_{0}$.

- We can suppose that $M=\mathbb{D}=\mathbb{D}_{1}$ and that $\lambda_{0}=0$ (here $\mathbb{D}=\mathbb{D}_{1}$ denotes the unit disc). Doing this, we actually prove a stronger statement, i.e., that $d d^{c} L \neq 0$ on every complex disc passing through $\lambda_{0}$ such that $\sigma(\lambda)$ is not contained in $f_{\lambda}^{n_{0}}(C)$ for every $\lambda$ is the selected disc. Moreover, we shall assume that $\mathcal{V}=\mathbb{D} \times V$.

- Without loss of generality, we can assume that $\sigma(\lambda)$ stays repelling for every $\lambda \in \mathbb{D}$. Up to considering an iterate of $f$, we can suppose that $\sigma(\lambda)$ is a repelling fixed point. Indeed, we can replace $n_{0}$ with $n_{0}+r$, for some $0 \leq r<n(\sigma)$, where $n(\sigma)$ is the period of $\sigma$, to ensure that now the new $n_{0}$ is a multiple of $n(\sigma)$.

- Using a local biholomorphism (change of coordinates), we can suppose that $\sigma(\lambda)$ is a constant in $V$, and we can assume that this constant is 0 .

- After possibly further rescaling, we can assume that $f^{n_{0}}(C)$ intersects $\{z=0\}$ only at $\lambda=0$.

- We denote by $B$ a small ball in $V$ centred at 0 .

Fix a $\delta$ such that $d_{k-1}^{*}<\delta<d_{t}$. Proposition 3.3 gives the existence of a ball $A_{0} \subset B$ and an $\eta$ such that the cylinder $T_{0}:=\mathbb{D}_{\eta} \times A_{0}$ admits at least $\delta^{N}$ inverse branches $h_{i}$ for $f^{N}$, with images contained in $T_{0}$. We explicitely notice that the images 
of $T_{0}$ under these inverse branches must be disjoint. Up to rescaling we can still assume that $\eta=1$.

The cylinder $T_{0}$ is naturally foliated by the "horizontal" holomorphic graphs $\Gamma_{\xi_{z}}$ 's, where $\xi_{z}(\lambda) \equiv z$, for $z \in A_{0}$. By construction, $T_{0}$ has at least $\delta^{N n}$ inverse branches for $f^{N n}$, with images contained in $T_{0}$. We denote these preimages by $T_{n, i}$, and we explicitely notice that every $T_{n, i}$ is biholomorphic to $T_{0}$, by the map $f^{N n}$. In particular, $f^{N n}$ induces a foliation on every $T_{n, i}$, given by the preimages of the $\Gamma_{\xi}$ z's by $f^{N n}$.

The following elementary lemma shows that (up to shrinking $B$ ) some component $\tilde{C}$ of $f^{n_{0}}(C)$ intersects the graph of every holomorphic map $\gamma: \mathbb{D} \rightarrow B$, and in particular every element of the induced foliation on $T_{n, i}$. This is a consequence of the fact that $f^{n_{0}}(C) \cap\{z=0\}=(0,0)$.

Lemma 3.5 Denote by $\mathcal{G}$ the set of holomorphic maps $\gamma: \mathbb{D} \rightarrow B$. Up to shrinking $B$, at least one irreducible component $\tilde{C}$ of $f^{n_{0}}(C)$ passing through $(0,0)$ intersects the graph of every element of $\mathcal{G}$.

Let $\tilde{C}$ be given by Lemma 3.5. In particular, $\tilde{C}$ intersects every element of the induced foliations on the $T_{n, i}$ 's. Let $B_{n, i}$ denote the intersection $T_{n, i} \cap \tilde{C}$ and set $D_{n, i}:=f^{N n}\left(B_{n, i}\right) \subset T_{0}$. The $D_{n, i}$ 's are non-empty analytic subsets of $T_{0}$ (since $f^{N n}: T_{n, i} \rightarrow T_{0}$ is a biholomorphism). Moreover, the graphs of the $\xi_{z}$ 's intersect every $D_{n, i}$, since their preimages in $T_{n, i}$ intersect every $B_{n, i}$. In particular, the projection of every $D_{n, i}$ on $V$ is equal to $A_{0}$.

Let us finally estimate the mass of $\left(f^{n_{0}+N n}\right)_{*}[C]$ on $\mathcal{U}_{\mathbb{D}}$. First of all, notice that $\left(f^{n_{0}}\right)_{*} \mathbf{C}_{f} \geq f_{*}^{n_{0}}[C] \geq\left[f^{n_{0}}(C)\right] \geq[\tilde{C}]$ as positive currents on $\mathcal{U}_{\mathbb{D}}$. This implies that

$$
\left\|f_{*}^{N n+n_{0}} \mathrm{C}_{f}\right\|_{\mathcal{U}_{\mathbb{D}}} \geq\left\|f_{*}^{N n}\left[f^{n_{0}}(C)\right]\right\|_{\mathcal{U}_{\mathbb{D}}} \geq\left\|f_{*}^{N n}[\tilde{C}]\right\|_{\mathcal{U}_{\mathbb{D}}} \geq\left\|f_{*}^{N n}[\tilde{C}]\right\|_{T_{0}} .
$$

Now, since $f^{N n}$ gives a biholomorphism from every $T_{n, i}$ to $T_{0}$ and all the $T_{n, i}$ 's are disjoint, we have

$$
\left\|f_{*}^{N n}[\tilde{C}]\right\|_{T_{0}} \geq\left\|f_{*}^{N n}\left(\sum_{i}\left[B_{n, i}\right]\right)\right\|_{T_{0}}=\sum_{i}\left\|f_{*}^{N n}\left[B_{n, i}\right]\right\|=\sum_{i}\left\|\left[D_{n, i}\right]\right\| .
$$

By Wirtinger formula, for every $n$ and $i$ the volume of $D_{n, i}$ is larger than the volume of its projection $A_{0}$ on $V$. Since by construction the last sum has at least $\delta^{N n}$ terms, we have

$$
\left\|f_{*}^{n_{0}+N n} \mathrm{C}_{f}\right\|_{\mathcal{U}_{\mathbb{D}}} \geq \operatorname{volume}\left(A_{0}\right) \cdot \delta^{N n}>\operatorname{volume}\left(A_{0}\right) \cdot\left(d_{k-1}^{*}\right)^{N n}
$$

and the assertion follows. 


\section{Dynamical stability of polynomial-like maps}

\subsection{Equilibrium webs}

We introduce here a notion of holomorphic motion for the equilibrium measures of a family of polynomial-like maps. Consider the space of holomorphic maps

$$
\mathcal{O}\left(M, \mathbb{C}^{k}, \mathcal{V}\right):=\left\{\gamma \in \mathcal{O}\left(M, \mathbb{C}^{k}\right): \forall \lambda \in M, \gamma(\lambda) \in V_{\lambda}\right\}
$$

endowed with the topology of local uniform convergence and its subset

$$
\mathcal{O}\left(M, \mathbb{C}^{k}, \mathcal{U}\right):=\left\{\gamma \in \mathcal{O}\left(M, \mathbb{C}^{k}\right): \forall \lambda \in M, \gamma(\lambda) \in U_{\lambda}\right\}
$$

By Montel Theorem, $\mathcal{O}\left(M, \mathbb{C}^{k}, \mathcal{U}\right)$ is relatively compact in $\mathcal{O}\left(M, \mathbb{C}^{k}, \mathcal{V}\right)$. The map $f$ induces an action $\mathcal{F}$ from $\mathcal{O}\left(M, \mathbb{C}^{k}, \mathcal{U}\right)$ to $\mathcal{O}\left(M, \mathbb{C}^{k}, \mathcal{V}\right)$ given by

$$
(\mathcal{F} \cdot \gamma)(\lambda)=f_{\lambda}(\gamma(\lambda))
$$

We now restrict ouselves to the subset $\mathcal{J}$ of $\mathcal{O}\left(M, \mathbb{C}^{k}, \mathcal{U}\right)$ given by

$$
\mathcal{J}:=\left\{\gamma \in \mathcal{O}\left(M, \mathbb{C}^{k}, \mathcal{U}\right): \gamma(\lambda) \in J_{\lambda} \text { for every } \lambda \in M\right\}
$$

This is an $\mathcal{F}$-invariant compact metric space with respect to the topology of local uniform convergence. Thus, $\mathcal{F}$ induces a well-defined dynamical system on it.

Nothing prevents the set $\mathcal{J}$ to be actually empty, but we have the following lemma (see [6]) which is a consequence of the lower semicontinuity of the Julia set (see [14]).

Lemma 4.1 Let $f: \mathcal{U} \rightarrow \mathcal{V}$ be a holomorphic family of polynomial-like maps of large topological degree and $\rho \in \mathcal{O}\left(M, \mathbb{C}^{k}, \mathcal{U}\right)$ such that $\rho(\lambda)$ is n-periodic for every $\lambda \in M$. Then, the set

$$
J_{\rho}:=\left\{\lambda \in M: \rho(\lambda) \text { is n-periodic, repelling and belongs to } J_{\lambda}\right\}
$$

is open.

Notice that a repelling cycle $\rho(\lambda)$ can leave the Julia set (i.e., the set $J_{\rho}$ is not necessarily closed). An example of this phenomenon is given in [6].

We denote by $p_{\lambda}: \mathcal{J} \rightarrow \mathbb{P}^{k}$ the evaluation map $\gamma \mapsto \gamma(\lambda)$. The map $\mathcal{F}: \mathcal{O}\left(M, \mathbb{C}^{k}, \mathcal{U}\right) \rightarrow \mathcal{O}\left(M, \mathbb{C}^{k}, \mathcal{V}\right)$ is proper. This follows from Montel Theorem since, for any $\lambda, p_{\lambda}$ is continuous and $f_{\lambda}: U_{\lambda} \rightarrow V_{\lambda}$ is proper. This means in particular that $\mathcal{F}$ induces a well defined notion of pushforward from the measures on $\mathcal{O}\left(M, \mathbb{C}^{k}, \mathcal{U}\right)$ to those on $\mathcal{O}\left(M, \mathbb{C}^{k}, \mathcal{V}\right)$.

Definition 4.2 Let $f: \mathcal{U} \rightarrow \mathcal{V}$ be a holomorphic family of polynomial-like maps. An equilibrium web is a probability measure $\mathcal{M}$ on $\mathcal{J}$ such that: 
1. $\mathcal{F}_{*} \mathcal{M}=\mathcal{M}$, and

2. $\left(p_{\lambda}\right)_{*} \mathcal{M}=\mu_{\lambda}$ for every $\lambda \in M$.

The equilibrium measures $\mu_{\lambda}$ move holomorphically (over $M$ ) if $f$ admits an equilibrium web.

Given an equilibrium web $\mathcal{M}$, we can see the triple $(\mathcal{J}, \mathcal{F}, \mathcal{M})$ as an invariant dynamical system. Moreover, we can associate to $\mathcal{M}$ the $(k, k)$-current on $\mathcal{U}$ given by $W_{\mathcal{M}}:=\int\left[\Gamma_{\gamma}\right] d \mathcal{M}(\gamma)$, where $\left[\Gamma_{\gamma}\right]$ denotes the integration current on the graph $\Gamma_{\gamma} \subset \mathcal{U}$ of the map $\gamma \in \mathcal{J}$. It is not difficult to check that $\mathcal{W}_{\mathcal{M}}$ is an equilibrium current for the family.

The next lemma gives some elementary properties of the support of any equilibrium web. The proof is the same as in the case of endomorphisms of $\mathbb{P}^{k}$ (see [5, Lemma 2.5]) and is thus omitted.

Lemma 4.3 Let $f: \mathcal{U} \rightarrow \mathcal{V}$ be a holomorphic family of polynomial-like maps of degree $d_{t} \geq 2$. Assume that $f$ admits an equilibrium web $\mathcal{M}$. Then

1. for every $\left(\lambda_{0}, z_{0}\right) \in M \times J_{\lambda_{0}}$ there exists an element $\gamma \in \operatorname{Supp} \mathcal{M}$ such that $z_{0}=\gamma(\lambda)$, and

2. for every $\left(\lambda_{0}, z_{0}\right) \in M \times J_{\lambda_{0}}$ such that $z_{0}$ is $n$-periodic and repelling for $f_{\lambda_{0}}$ there exists a unique $\gamma \in \operatorname{Supp} \mathcal{M}$ such that $z_{0}=\gamma\left(\lambda_{0}\right)$ and $\gamma(\lambda)$ is n-periodic for $f_{\lambda}$ for every $\lambda \in M$. Moreover, $\gamma\left(\lambda_{0}\right) \neq \gamma^{\prime}\left(\lambda_{0}\right)$ for every $\gamma^{\prime} \in \operatorname{Supp} \mathcal{M}$ different from $\gamma$.

The following theorem allows us to construct equilibrium webs starting from particular elements in $\mathcal{O}\left(M, \mathbb{C}^{k}, \mathcal{U}\right)$. The proof is analogous to the one on $\mathbb{P}^{k}$ (see [5]) and is based on Theorem 2.8. We refer to [6] for the details in this setting. We just notice that the assumption on the parameter space to be simply connected in the second point is needed to ensure the existence of the preimages.

Theorem 4.4 Let $f: \mathcal{U} \rightarrow \mathcal{V}$ be a holomorphic family of polynomial-like maps of large topological degree $d_{t} \geq 2$.

1. Assume that the repelling $J$-cycles of $f$ asymptotically move holomorphically over the parameter space $M$ and let $\left(\rho_{n, j}\right)_{1 \leq j \leq N_{n}}$ be the elements of $\mathcal{J}$ given by the motions of these cycles. Then, the equilibrium measures move holomorphically and any limit of $\left(\frac{1}{d_{t}^{n}} \sum_{j=1}^{N_{n}} \delta_{\rho_{n, j}}\right)_{n}$ is a equilibrium web.

2. Assume that the parameter space $M$ is simply connected and that there exists $\gamma \in \mathcal{O}\left(M, \mathbb{C}^{k}, \mathcal{U}\right)$ whose graph $\Gamma_{\gamma}$ does not intersect the post-critical set of $f$. Then, the equilibrium measures move holomorphically and any limit of $\left(\frac{1}{n} \sum_{l=1}^{n} \frac{1}{d_{t}^{l}} \sum_{\mathcal{F}^{l} \sigma=\gamma} \delta_{\sigma}\right)_{n}$ is an equilibrium web.

\subsection{A preliminary characterization of stability}

The following Theorem shows the equivalence of the conditions A.3 and A.4 in Theorem C. 
Theorem 4.5 Let $f: \mathcal{U} \rightarrow \mathcal{V}$ be a holomorphic family of polynomial-like maps of large topological degree $d_{t} \geq 2$. Then the following are equivalent:

I.1 for every $\lambda_{0} \in M$ there exists a neighbourhood $M_{0} \Subset M$ where the measures $\mu_{\lambda}$ move holomorphically and $f$ admits an equilibrium web $\mathcal{M}=\lim _{n} \mathcal{M}_{n}$ such that the graph of any $\gamma \in \cup_{n} \operatorname{Supp} \mathcal{M}_{n}$ avoids the critical set of $f$;

I.2 the function $L$ is pluriharmonic on $M$;

I.3 there are no Misiurewicz parameters in $M$;

I.4 for every $\lambda_{0} \in M$ there exists a neighbourhood $M_{0} \Subset M$ and a holomorphic map $\gamma \in \mathcal{O}\left(M_{0}, \mathbb{C}^{k}, \mathcal{U}\right)$ whose graph does not intersect the postcritical set of $f$.

Theorem 4.4 readily proves that I.4 imples I.1, while Theorem A gives the implication I.2 $\Rightarrow$ I.3. The strategy for the two implications I. $1 \Rightarrow$ I.2 and I.3 $\Rightarrow$ I.4 follows the same lines of the one on $\mathbb{P}^{k}$. In particular, for the first one the only small difference is how to get an estimate (Hölder in $\varepsilon$ ) for the $\mu$-measure of an $\varepsilon$-neighbourhood of an analytic set. In the case of endomorphisms of $\mathbb{P}^{k}$, this follows from the Hölder continuity of the potential of the Green current. Here we can exploit the fact that, for every psh function $u$, the function $e^{|u|}$ is integrable with respect to the equilibrium measure of a polynomial-like map of large topological degree ([14, Theorem 2.34]). We refer to [6] for a complete proof.

For what concerns the last missing implication the proof can be reduced, (as in the case of endomorphisms of $\mathbb{P}^{k}$ ), by means of Hurwitz Theorem, to the proof of the existence (Theorem 4.6) of a hyperbolic set satisfying certain properties (see [6]).

Theorem 4.6 Let $f: \mathcal{U} \rightarrow \mathcal{V}$ be a holomorphic family of polynomial-like maps of large topological degree $d_{t}$. Then there exists an integer $N$, a compact hyperbolic set $E_{0} \subset J_{\lambda_{0}}$ for $f_{\lambda_{0}}^{N}$ and a continuous holomorphic motion $h: B_{r} \times E_{0} \rightarrow \mathbb{C}^{k}$ (defined on some small ball $B_{r}$ of radius $r$ and centered at $\lambda_{0}$ ) such that:

1. the repelling periodic points of $f_{\lambda_{0}}^{N}$ are dense in $E_{0}$ and $E_{0}$ is not contained in the postcritical set of $f_{\lambda_{0}}^{N}$;

2. $h_{\lambda}\left(E_{0}\right) \in J_{\lambda}$ for every $\lambda \in B_{r}$;

3. if $z$ is periodic repelling for $f_{\lambda_{0}}^{N}$ then $h_{\lambda}(z)$ is periodic and repelling for $f_{\lambda}^{N}$.

To prove Theorem 4.6 on $\mathbb{P}^{k}$, one needs to ensure that a hyperbolic set of sufficiently large entropy cannot be contained in the postcritical set and must, on the other hand, be contained in the Julia set. In our setting, the analogue of the first propery is given by Lemma 4.8 below, which is a direct consequence of Lemma 4.7, combined with a relative version of the Variational principle.

Lemma 4.7 Let $f: U \rightarrow V$ be a polynomial like map of topological degree $d_{t}$. Let $K$ be the filled Julia set, $X$ an analytic subset of $V$ of dimension $p$, and $\delta_{n}$ be such that $\left\|f_{*}^{n}[X]\right\|_{U} \leq \delta_{n}$. Then

$$
h_{t}(f, K \cap X) \leq \limsup _{n \rightarrow \infty} \frac{1}{n} \log \delta_{n} \leq d_{p}^{*} .
$$


This lemma is proved by following the strategy used by Gromov [19] to estimate the topological entropy of endomorphisms of $\mathbb{P}^{k}$, and adapted by Dinh and Sibony $[12,14]$ to the polynomial-like setting. Since only minor modifications are needed, we refer to [6] for a complete proof.

Lemma 4.8 Let $g$ be a polynomial-like map of large topological degree. Let $v$ be an ergodic invariant probability measure for $g$ whose metric entropy $h_{v}$ satisfies $h_{v}>\log d_{p}^{*}$. Then, $v$ gives no mass to analytic subsets of dimension $\leq p$.

The second problem (i.e., ensuring that the hyperbolic set stays inside the Julia set) will be adressed by means of the following lemma.

Lemma 4.9 (see also [15], Lemma 2.3) Let $f: \mathcal{U} \rightarrow \mathcal{V}$ be a holomorphic family of polynomial-like maps with parameter space $M$. Let $E_{0}$ be a hyperbolic set for $f_{\lambda_{0}}$ contained in $J_{\lambda_{0}}$, such that repelling periodic points are dense in $E_{0}$ and $\left\|\left(d f_{\lambda}\right)^{-1}\right\|^{-1}>K>3$ on a neighbourhood of $\left(E_{0}\right)_{\tau}$ in the product space. Let $h$ be a continuous holomorphic motion of $E_{0}$ as a hyperbolic set on some ball $B \subset M$, preserving the repelling cycles. Then $h_{\lambda}\left(E_{0}\right)$ is contained in $J_{\lambda}$, for $\lambda$ sufficiently close to $\lambda_{0}$.

Proof We denote by $\gamma_{z}$ the motion of a point $z \in E_{0}$ as part of the given holomorphic motion of the hyperbolic set.

First of all, notice that repelling points must be dense in $E_{\lambda}$ for every $\lambda$, by the continuity of the motion and the fact that they are preserved by it. Moreover, by Lemma 4.1, every repelling cycle stays in $J_{\lambda}$ for $\lambda$ in a neighbourhood of $\lambda_{0}$. It is thus enough to ensure that this neighbourhood can be taken uniform for all the cycles.

Since $\left\|d f_{\lambda}^{-1}\right\|^{-1}>3$ on a neighbourhood $\left(E_{0}\right)_{\tau}$ of $E_{0}$ in the product space, we can restrict ourselves to $\lambda \in B\left(\lambda_{0}, \tau\right)$ and so assume that $\left\|d f_{\lambda}^{-1}\right\|^{-1}>3$ on a $\tau$ neighbourhood of every $z \in E_{\lambda}$, for every $\lambda$. Moreover, since the set of motions $\gamma_{z}$ of points in $E_{0}$ is compact (by continuity), by shrinking $\tau$ we can assume that $\gamma_{z}(\lambda) \in B(z, \tau / 10)$ for every $z \in E_{0}$ and $\lambda$. Finally, by the lower semicontinuity of the Julia set ([14]), up to shrinking again the parameter space we can assume that $J_{\lambda_{0}} \subset\left(J_{\lambda}\right)_{\tau / 10}$ for every $\lambda$. These two assumptions imply that, for every $\lambda$ and every $z \in E_{\lambda}$, there exists at least a point of $J_{\lambda}$ in the ball $B(z, \tau / 2)$. Consider now any $n$-periodic repelling point $p_{0}$ in $E_{\lambda}$ for $f_{\lambda}$, and let $\left\{p_{i}\right\}=\left\{f_{\lambda}^{i}\left(p_{0}\right)\right\}$ be its cycle (and thus with $p_{0}=p_{n}$ ). Fix a point $z_{0} \in J_{\lambda} \cap B\left(p_{0}, \tau\right)$. By hyperbolicity (and since without loss of generality we can assume that $\left.\tau \leq\left(1+\sup _{B_{\tau}}\left\|f_{\lambda}\right\|_{C^{2}}\right)^{-1}\right)$, every ball $B\left(p_{i}, \tau\right)$ has an inverse branch for $f_{\lambda}$ defined on it, with image strictly contained in the ball $B\left(p_{i-1}, \tau\right)$ and strictly contracting. This implies that there exists an inverse branch $g_{0}$ for $f_{\lambda}^{n}$ of $B\left(p_{0}, \tau\right)$, strictly contracting and with image strictly contained in $B\left(p_{0}, \tau\right)$ (and containing $p_{0}$ ). So, a sequence of inverse images of $z_{0}$ for $f_{\lambda}$ must converge to $p_{0}$, and so $p_{0} \in J_{\lambda}$. The assertion is proved.

Proof (Proof of Theorem 4.6) First of all, we need the hyperbolic set $E_{0}$. By Lemma 3.4, we can take a closed ball $A$, a constant $0<\rho<1$ and a sufficiently large $N$ such that the cardinality $N^{\prime}$ of $C_{N}(A, \rho)$ (see (3.2)) satisfies $N^{\prime} \geq\left(d_{k-1}^{*}\right)^{N}$ 
(since by assumption $d_{k-1}^{*}<d_{t}$ ). We then consider the set $E_{0}$ given by the intersection $E_{0}=\cap_{k \geq 0} E_{k}$, where $E_{k}$ is given by

$$
E_{k}:=\left\{g_{i_{1}} \circ \cdots \circ g_{i_{k}}(A):\left(i_{1}, \ldots, i_{k}\right) \in\left\{1, \ldots, N^{\prime}\right\}^{k}\right\}
$$

where the $g_{i}$ 's are the elements of $C_{N}(A, \rho)$. The set $E_{0}$ is then hyperbolic, and contained in $J_{0}$ (since $A \cap J \neq \emptyset$, every point in $E_{0}$ is accumulated by points in the Julia set). Moreover, repelling cycles (for $f_{0}^{N}$ ) are dense in $E_{0}$.

Let $\Sigma:\left\{1, \ldots, N^{\prime}\right\}^{\mathbb{N}^{*}}$ and fix a point $z \in E_{0}$. Notice that the map $\omega: \Sigma \rightarrow E_{0}$ given by $\omega\left(i_{1}, i_{2}, \ldots\right)=\lim _{k \rightarrow \infty} g_{i_{1}} \circ \cdots \circ g_{i_{k}}(z)$ satisfies the relation $f^{N} \circ \omega=\omega \circ s$, where $s$ denotes the left shift $\left(i_{1}, \ldots, i_{k}, \ldots\right) \stackrel{s}{\mapsto}\left(i_{2}, \ldots, i_{k+1}, \ldots\right)$. We can thus pushforward with $\omega$ the uniform product measure on $\Sigma$. Since this is an $s$-invariant ergodic measure, its pushforward $v$ is an $f^{N}$-invariant ergodic measure on $E_{0} \subset J_{0}$. The metric entropy of $v$ thus satisfies $h_{v} \geq \log N^{\prime} \geq \log \left(d_{k-1}^{*}\right)^{N}$, and this implies (by Lemma 4.8) that $v$ gives no mass to analytic subsets. In particular, $E_{0}$ is not contained in the postcritical set of $f_{0}$.

We need to prove the points 2 and 3 in the theorem. It is a classical result (see [5, Appendix A.1]) that $E_{0}$ admits a continuous holomorphic motion that preserves the repelling cycles, and thus 3 follows. The second point then follows from Lemma 4.9 (and the density of the repelling cycles in $E_{0}$ ).

Once we have established the existence of a hyperbolic set as in Theorem 4.6, the proof of the implication I.3 $\Rightarrow$ I.4 is the same as on $\mathbb{P}^{k}($ see $[5,6])$.

\subsection{Holomorphic motions}

The following Theorem 4.11 implies Theorem B, and thus the equivalence between the conditions A.1 and A.2 in Theorem C. We need the following definition.

Definition 4.10 An equilibrium web $\mathcal{M}$ is acritical if $\mathcal{M}\left(\mathcal{J}_{s}\right)=0$, where

$$
\mathcal{J}_{s}:=\left\{\gamma \in \mathcal{J}: \Gamma_{\gamma} \cap\left(\bigcup_{m, n \geq 0} f^{-m}\left(f^{n}\left(C_{f}\right)\right)\right) \neq \varnothing\right\} .
$$

Theorem 4.11 Let $f: \mathcal{U} \rightarrow \mathcal{V}$ be a holomorphic family of polynomial-like maps of large topological degree $d_{t} \geq 2$. Assume that the parameter space is simply connected. Then the following are equivalent:

II.1 asymptotically all J-cycles move holomorphically;

II.2 there exists an acritical equilibrium web $\mathcal{M}$;

II.3 there exists an equilibrium lamination for $f$.

Moreover, if the previous conditions hold, the system admits a unique equilibrium web, which is ergodic and mixing. 
As we mentioned in the introduction, we shall only show how to recover the asymptotic holomorphic motion of the repelling cycles from the two conditions II.3 and II.2. Indeed, the construction of an equilibrium lamination starting from an acritical web is literally the same as in the case of $\mathbb{P}^{k}$. The crucial point in that proof is establishing the following backward contraction property (Proposition 4.12) (which is actually used in both the implications II.2 $\Rightarrow$ II.3 $\Rightarrow$ II.1). We set $\mathcal{X}:=\mathcal{J} \backslash \mathcal{J}_{s}$ (notice that this is a full measure subset for an acritical web) and let $(\widehat{\mathcal{X}}, \widehat{\mathcal{F}}, \widehat{\mathcal{M}})$ be the natural extension (see [9]) of the system $(\mathcal{X}, \mathcal{F}, \mathcal{M})$, i.e., the set of the histories of elements of $\mathcal{X}$

$$
\widehat{\gamma}:=\left(\ldots, \gamma_{-j}, \gamma_{-j+1}, \ldots, \gamma_{-1}, \gamma_{0}, \gamma_{1}, \ldots\right)
$$

where $\mathcal{F}\left(\gamma_{-j}\right)=\gamma_{-j+1}$. The map $\mathcal{F}$ lifts to a bijection $\widehat{\mathcal{F}}$ given by

$$
\widehat{\mathcal{F}}(\widehat{\gamma}):=\left(\ldots \mathcal{F}\left(\gamma_{-j}\right), \mathcal{F}\left(\gamma_{-j+1}\right) \ldots\right)
$$

and thus correspond to the shift operator. $\widehat{\mathcal{M}}$ is the only measure on $\widehat{\mathcal{X}}$ such that $\left(\pi_{j}\right)_{\star}(\widehat{\mathcal{M}})=\mathcal{M}$ for any projection $\pi_{j}: \widehat{\mathcal{X}} \rightarrow \widehat{\mathcal{X}}$ given by $\pi_{j}(\widehat{\gamma})=\gamma_{j}$. When $\mathcal{M}$ is ergodic (or mixing), the same is true for $\widehat{\mathcal{M}}$.

Given $\gamma \in \mathcal{X}$, denote by $f_{\gamma}$ the injective map which is induced by $f$ on some neighbourhood of the graph $\Gamma_{\gamma}$ and by $f_{\gamma}^{-1}$ the inverse branch of $f_{\gamma}$, which is defined on some neighbourhood of $\Gamma_{\mathcal{F}(\gamma)}$. Given $\widehat{\gamma} \in \widehat{\mathcal{X}}$ and $n \in \mathbb{N}$ we thus define the iterated inverse branch $f_{\widehat{\gamma}}^{-n}$ of $f$ along $\widehat{\gamma}$ and of depth $n$ by

$$
f_{\widehat{\gamma}}^{-n}:=f_{\gamma_{-n}}^{-1} \circ \cdots \circ f_{\gamma_{-2}}^{-1} \circ f_{\gamma_{-1}}^{-1}
$$

Given $\eta>0$, we shall denote by $T_{M_{0}}\left(\gamma_{0}, \eta\right)$ the tubular neighbourhood

$$
T_{M_{0}}\left(\gamma_{0}, \eta\right):=\left\{(\lambda, z) \in M_{0} \times \mathbb{C}^{k}: d\left(z, \gamma_{0}(\lambda)\right)<\eta\right\}
$$

Proposition 4.12 Let $f: \mathcal{U} \rightarrow \mathcal{V}$ be a holomorphic family of polynomial-like maps over $M$ of large topological degree $d \geq 2$ which admits an acritical and ergodic equilibrium web $\mathcal{M}$. Then (up to an iterate) there exist a Borel subset $\widehat{\mathcal{Y}} \subset \widehat{\mathcal{X}}$ such that $\widehat{\mathcal{M}}(\widehat{\mathcal{Y}})=1$, a measurable function $\widehat{\eta}: \widehat{\mathcal{Y}} \rightarrow] 0,1]$ and a constant $A>0$ which satisfy the following properties.

For every $\widehat{\gamma} \in \widehat{\mathcal{Y}}$ and every $n \in \mathbb{N}$ the iterated inverse branch $f_{\widehat{\gamma}}^{-n}$ is defined on the tubular neighbourhood $T_{U_{0}}\left(\gamma_{0}, \widehat{\eta}_{p}(\widehat{\gamma})\right)$ of $\Gamma_{\gamma_{0}} \cap\left(U_{0} \times \mathbb{C}^{k}\right)$ and

$$
f_{\widehat{\gamma}}^{-n}\left(T_{U_{0}}\left(\gamma_{0}, \widehat{\eta}_{p}(\widehat{\gamma})\right)\right) \subset T_{U_{0}}\left(\gamma_{-n}, e^{-n A}\right)
$$

Moreover, the map $f_{\widehat{\gamma}}^{-n}$ is Lipschitz with Lip $f_{\widehat{\gamma}}^{-n} \leq \widehat{l}_{p}(\widehat{\gamma}) e^{-n A}$ where $\widehat{l}_{p}(\widehat{\gamma}) \geq 1$ is a function on $\widehat{\mathcal{Y}}$.

Notice that this is essentially a local statement on the parameter space. The assumption on the family to be of large topological degree is crucial here to ensure that all 
Lyapunov exponents are positive. Once Proposition 4.12 is established, the implication II. $2 \Rightarrow$ II.3 follows by an application of Poincaré recurrence theorem. Moreover, the uniqueness of the equilibrium web and its mixing behaviour also easily follow.

The fact that either the asymptotic motion of the repelling cycles or the existence of an equilibrium lamination implies the existence of an ergodic acritical web is again proved in same exact way than on $\mathbb{P}^{k}$.

Proposition 4.13 Let $f: \mathcal{U} \rightarrow \mathcal{V}$ be a holomorphic family of polynomial-like maps of large topological degree $d_{t} \geq 2$. Assume that one of the following holds:

1. asymptotically all repelling $J$-cycles move holomorphically, or

2. there exists a holomorphic map $\gamma \in \mathcal{O}\left(M, \mathbb{C}^{k}, \mathcal{V}\right)$ such that $\Gamma_{\gamma}$ does not intersect the postcritical set of $f$.

Then $f$ admits an ergodic acritical equilibrium web.

The important points in the proof are the following: the equilibrium measure cannot charge the postcritical set (Theorem 2.5) and we can build an equilibrium web (by means of Theorem 4.4) satisfying the assumptions of Theorem 4.5(I.1) (which implies that the family has no Misiurewicz parameters).

We are thus left to prove that the two (equivalent) conditions II. 3 and II. 2 imply the asymptotic motion of the repelling points. We stress that, in order to do this, we do not need to make any further assumption on the family we are considering. We start noticing that just the existence of any equilibrium web implies the existence of a set $\mathcal{P} \subset \mathcal{J}$ satisfying all the properties required by Definition 1.3 but the last one. This is an immediate consequence of Lemma 4.3.

Lemma 4.14 Let $f$ be a holomorphic family of polynomial-like maps of large topologicald degree $d_{t}$. Assume that there exists an equilibrium web $\mathcal{M}$ for $f$. Then there exists a subset $\mathcal{P}=\cup_{n} \mathcal{P}_{n} \subset \mathcal{J}$ such that

1. $\operatorname{Card} \mathcal{P}_{n}=d_{t}^{n}+o\left(d_{t}^{n}\right)$;

2. every element in $\mathcal{P}_{n}$ is n-periodic;

3. we have $\sum_{\gamma \in \mathcal{P}_{n}} \delta_{\gamma} \rightarrow \mathcal{M}^{\prime}$, where $\mathcal{M}^{\prime}$ is a (possibly different) equilibrium web.

Notice that, if the equilibrium web $\mathcal{M}$ in the statement is acritical, by the uniqueness recalled above we have $\mathcal{M}=\mathcal{M}^{\prime}$.

Proof Let us fix $\lambda_{0}$ in the parameter space. Since $f_{\lambda_{0}}$ has large topological degree, Theorem 2.8 gives $d_{t}^{n}+o\left(d_{t}^{n}\right)$ repelling periodic points for $f_{\lambda_{0}}$ contained in the Julia set $J_{\lambda_{0}}$. By Lemma 4.3(2), for every such point $p$ of period $n$ there exists an element $\gamma_{p} \in \mathcal{J}$ such that $\gamma_{p}\left(\lambda_{0}\right)=p$ and $\mathcal{F}^{n}\left(\gamma_{p}\right)=\gamma_{p}$. This gives the first two assertions of the statement. The last one follows by Theorem 4.4 .

In order to recover the asymptotic motion of the repelling cycles as in Definition 1.3, we thus just need to prove that, on any $M^{\prime} \Subset M$, asymptotically all $\gamma_{p} \in \mathcal{P}_{n}$ given by Lemma 4.14 are repelling. This will be done by means of the following general lemma, which allows us to recover the existence of repelling points for a dynamical system from the information about backward contraction of balls along negative orbits. 
This can be seen as a generalization of a classical strategy [8] (see also [3]). We keep the notations introduced before Proposition 4.12 regarding the natural extension of a dynamical system and the inverse branches along negative orbits.

Lemma 4.15 Let $\mathcal{F}: \mathcal{K} \rightarrow \mathcal{K}$ be a continuous map from a compact metric space $\mathcal{K}$ to itself. Assume that, for every $n$, the number of periodic repelling points of period dividing $n$ is less than $d^{n}+o\left(d^{n}\right)$ for some integer $d \geq 2$. Let $v$ be a probability measure on $\mathcal{K}$ which is invariant, mixing and of constant Jacobian $d$ for $\mathcal{F}$.

Suppose that there exists an $\mathcal{F}$-invariant subset $\mathcal{L} \subset \mathcal{K}$ such that $v(\mathcal{L})=1$ and $\mathcal{F}: \mathcal{L} \rightarrow \mathcal{L}$ is a covering of degree $d$. Assume moreover that the natural extension $(\widehat{\mathcal{L}}, \widehat{\mathcal{F}}, \widehat{v})$ of the induced system $(\mathcal{L}, \mathcal{F}, v)$ satisfies the following properties.

(P1) For every $\widehat{x} \in \widehat{\mathcal{L}}$ the inverse branch $\mathcal{F}_{\widehat{x}}^{-n}$ is defined and Lipschitz on the open ball $B\left(x_{0}, \eta(\widehat{x})\right)$ with $\operatorname{Lip}\left(\mathcal{F}_{\widehat{x}}^{-n}\right) \leq l(\widehat{x}) e^{-n L}$, for some positive measurable functions $\eta$ and $l$ and some positive constant $L$.

(P2) $\forall x_{0} \in \mathcal{L}, \forall N$, for every closed subset $C$ of $B\left(x_{0}, \frac{1}{N}\right)$ the preimages $\mathcal{F}_{\widehat{x}}^{-n}(C)$ with $\eta(\widehat{x})>\frac{1}{N}$ (well defined by $P 1$ ) are sent bijectively to $C$ by $\mathcal{F}^{n}$. For $n$ large enough, they are disjoint.

Then,

$$
\frac{1}{d^{n}} \sum_{p \in R_{n}} \delta_{p} \rightarrow v
$$

where $R_{n}$ is the set of all repelling periodic points of period (dividing) $n$.

By repelling periodic point here we mean the following: a point $x_{0}$ such that, for some $n, \mathcal{F}^{n}\left(x_{0}\right)=x_{0}$ and there exists a local inverse branch $\mathcal{H}$ for $\mathcal{F}^{n}$ sending $x_{0}$ to $x_{0}$ and such that $\operatorname{Lip} \mathcal{H}_{x_{0}}<1$.

Proof We let $\tilde{\sigma}$ be any limit value of the sequence $\sigma_{n}:=\frac{1}{d^{n}} \sum_{p \in R_{n}} \delta_{p}$. Remark that

$$
\tilde{\sigma}(\mathcal{K}) \leq \limsup _{n \rightarrow \infty} \sigma_{n}(\mathcal{K}) \leq \lim _{n \rightarrow \infty} \frac{d^{n}+o\left(d^{n}\right)}{d^{n}}=1
$$

For every $N \in \mathbb{N}$, let $\widehat{\mathcal{L}}_{N} \subset \widehat{\mathcal{L}}$ be defined as

$$
\widehat{\mathcal{L}}_{N}=\left\{\widehat{x}: \eta(\widehat{x})>\frac{1}{N} \text { and } l(\widehat{x}) \leq N\right\}
$$

and set $\widehat{v}_{N}:=1_{\widehat{\mathcal{L}}_{N}} \widehat{v}$ and $v_{N}=\left(\pi_{0}\right)_{*} \widehat{v}_{N}$. We also set $\mathcal{L}_{N}:=\pi_{0}\left(\widehat{\mathcal{L}}_{N}\right)$. We are going to prove that

$$
\tilde{\sigma}(A) \geq v_{N}(A) \text { for every borelian } A, \quad \forall N \in \mathbb{N} \text {. }
$$

As by hypothesis $v_{N}(A) \rightarrow v(A)$ as $N \rightarrow \infty$, the assertion will then follow from (4.1) and (4.2). So we turn to prove (4.2). In order to do this, it suffices to prove the following: 
$\forall N \in \mathbb{N}, \forall \widehat{a} \in \widehat{\mathcal{L}}_{N}$, for every closed subset $C$ of $B\left(a_{0}, \frac{1}{2 N}\right): \tilde{\sigma}(C) \geq v_{N}(C)$.

Indeed, given any Borelian subset $A \subset \mathcal{K}$, since $\mathcal{K}$ is compact we can find a partition of $A \cap \mathcal{L}_{N}$ into finite borelian sets $A_{i}$, each of which contained in an open ball $B\left(a_{0}^{i}, \frac{1}{3 N}\right)$, with $\widehat{a}^{i} \in \mathcal{L}_{N}$. The assertion thus follows from (4.3) since, for every $A_{i}$, the values $\tilde{\sigma}\left(A_{i}\right)$ and $v_{N}\left(A_{i}\right)$ are the suprema of the respective measures on closed subsets of $A_{i}$ (which by construction are contained in $B\left(a_{0}^{i}, \frac{1}{2 N}\right)$ ).

In the following we thus fix a closed subset $C \subset B\left(a_{0}, \frac{1}{2 N}\right)$. We shall denote by $C_{\delta}$ the closed $\delta$-neighbourhood of $C$ (in $\mathcal{K}$ ). Take some $\delta$ such that $\delta<\frac{1}{2 N}$ and notice that, since $\widehat{a} \in \widehat{\mathcal{L}}_{N}$, we have $C_{\delta} \subset B\left(a_{0}, \frac{1}{N}\right) \subset B\left(a_{0}, \eta(\widehat{a})\right)$. Then, according to the property (1) of the natural extension $(\widehat{\mathcal{L}}, \widehat{\mathcal{F}}, \widehat{v})$, we can define the set:

$$
\widehat{R}_{n}^{\delta}=\left\{\widehat{x} \in \widehat{C}_{\delta} \cap \widehat{\mathcal{L}}_{N}: x_{0}=a_{0} \text { and } \mathcal{F}_{\widehat{x}}^{-n}\left(C_{\delta}\right) \cap C \neq \emptyset\right\} .
$$

Let us denote by $S_{n}^{\delta}$ the set of preimages of $C_{\delta}$ of the form $\mathcal{F}_{\widehat{x}}^{-n}\left(C_{\delta}\right)$ with $\widehat{x} \in \widehat{R}_{n}^{\delta}$, by the property (2) of $(\widehat{\mathcal{L}}, \widehat{\mathcal{F}}, \widehat{v})$, the elements of $S_{n}^{\delta}$ are mutually disjoint for $n \geq \tilde{n}_{0}$ (and of course Card $S_{n}^{\delta} \leq d^{n}$ ). We claim that Card $S_{n}^{\delta}$ satisfies the following two estimates:

1. $\frac{1}{d^{n}}$ Card $S_{n}^{\delta} \leq \sigma_{n}\left(C_{\delta}\right)$, for $n \geq n_{0} \geq \tilde{n}_{0}$, where $n_{0}$ depends only on $C$ and $\delta$;

2. $\frac{1}{d^{n}} \operatorname{Card} S_{n}^{\delta} v\left(C_{\delta}\right) \geq \widehat{v}\left(\widehat{\mathcal{F}}^{-n}\left(\widehat{C}_{\delta} \cap \widehat{\mathcal{L}}_{N}\right) \cap \widehat{C}\right)$.

Before proving the estimates (1) and (2), let us show how (4.3) follows from them. Combining (1) and (2) we get $\widehat{v}\left(\widehat{\mathcal{F}}^{-n}\left(\widehat{C}_{\delta} \cap \widehat{\mathcal{L}}_{N}\right) \cap \widehat{C}\right) \leq v\left(C_{\delta}\right) \sigma_{n}\left(C_{\delta}\right)$ and, since $\widehat{v}$ is mixing, letting $n \rightarrow \infty$ on a subsequence such that $\sigma_{n_{i}} \rightarrow \tilde{\sigma}$ we find $\widehat{v}\left(\widehat{C}_{\delta} \cap \widehat{\mathcal{L}}_{N}\right) \widehat{v}(\widehat{C}) \leq v\left(C_{\delta}\right) \tilde{\sigma}\left(C_{\delta}\right)$. Since the left hand side is equal to $v_{N}\left(C_{\delta}\right) v(C)$ (and $C$ is closed), (4.3) follows letting $\delta \rightarrow 0$.

We are thus left to proving the inequalities (1) and (2) above. We shall see that the first one follows from the Lipschitz estimate on $\mathcal{F}_{\widehat{x}}^{-n}$, while the second is a consequence of the fact that $v$ is of constant Jacobian (i.e., for every borelian set $A \subset \mathcal{K}$ on which $\mathcal{F}$ is injective we have $d v(A)=v(\mathcal{F}(A)))$.

Let us start with (1). We have to find an integer $n_{0}$ such that, for $n \geq n_{0}$, the neighbourhood $C_{\delta}$ contains al least Card $S_{n}^{\delta}$ repelling periodic points for $\mathcal{F}$. Take any $\widehat{x} \in \widehat{R}_{n}^{\delta}$. Since $\widehat{R}_{n}^{\delta} \subset \widehat{\mathcal{L}}_{N}$, one has $\eta(\widehat{x}) \geq \frac{1}{N}$ and $l(\widehat{x}) \leq N$. This means that $\mathcal{F}_{\widehat{x}}^{-n}$ is well defined on $C_{\delta} \subset B\left(a_{0}, \frac{1}{N}\right)$ and that $\operatorname{Diam} \mathcal{F}_{\widehat{x}}^{-n}\left(C_{\delta}\right) \leq \frac{1}{N} \operatorname{Lip} \mathcal{F}_{\widehat{x}}^{-n} \leq$ $\frac{1}{N} N e^{-n L}=e^{-n L}$. Let us now take $n_{0}$ such that $3 e^{-n_{0} L}<\delta$. Since, by definition of $\widehat{R}_{n}^{\delta}$, we have that $\mathcal{F}_{\widehat{x}}^{-n}\left(C_{\delta}\right)$ intersects $C$ and $C \subset C_{\delta}$, it follows that $\mathcal{F}_{\widehat{x}}^{-n}\left(C_{\delta}\right) \subset C_{\delta}$ for every $\widehat{x} \in \widehat{R}_{n}^{\delta}$, with $n \geq n_{0}$. So, since $C_{\delta}$ is itself a compact metric space and $\mathcal{F}_{\widehat{x}}^{-n}$ is stricly contracting on it (the condition $3 e^{-n_{0} L}<\delta<\frac{1}{2 N}$ also implies that $\operatorname{Lip} \mathcal{F}_{\hat{x}}^{-n}<1$ for $n \geq n_{0}$ ), we find a (unique) fixed point for it in $C_{\delta}$. Since the elements of $S_{n}^{\delta}$ are disjoint, we have found at least Card $S_{n}^{\delta}$ periodic points (whose 
period divides $n$ ) for $\mathcal{F}$ in $C_{\delta}$, which must be repelling by the Lipschitz estimate of the local inverse, and so (1) is proved.

For the second inequality, we have

$$
\begin{aligned}
& \widehat{v}\left(\widehat{\mathcal{F}}^{-n}\left(\widehat{C}_{\delta} \cap \widehat{\mathcal{L}}_{N}\right) \cap \widehat{C}\right) \leq v\left(\pi\left(\widehat{\mathcal{F}}^{-n}\left(\widehat{C}_{\delta} \cap \widehat{\mathcal{L}}_{N}\right) \cap \widehat{C}\right)\right) \\
& \leq v\left(\bigcup_{\widehat{x} \in \widehat{R}_{n}^{\delta}}\left(\mathcal{F}_{\widehat{x}}^{-n}\left(C_{\delta}\right)\right) \cap C\right) \leq v\left(\bigcup_{\widehat{x} \in \widehat{R}_{n}^{\delta}}\left(\mathcal{F}_{\widehat{x}}^{-n}\left(C_{\delta}\right)\right)\right) \\
& =\sum_{\mathcal{F}_{\widehat{x}}^{-n}\left(C_{\delta}\right) \in S_{n}^{\delta}} v\left(\mathcal{F}_{\widehat{x}}^{-n}\left(C_{\delta}\right)\right)=\sum_{\mathcal{F}_{\widehat{x}}^{-n}\left(C_{\delta}\right) \in S_{n}^{\delta}} \frac{1}{d^{n}} v\left(C_{\delta}\right)=\frac{1}{d^{n}}\left(\text { Card } S_{n}^{\delta}\right) v\left(C_{\delta}\right)
\end{aligned}
$$

where the second equality follows from the fact that $v$ is of constant Jacobian.

We can now show how conditions II.2 and II.3 (which we recall are equivalent) imply condition II.1 in Theorem 4.11. Recall that this also proves Theorem B.

Theorem 4.16 Let $f: \mathcal{U} \rightarrow \mathcal{V}$ be a holomorphic family of polynomial-like maps, of degree $d_{t} \geq 2$. Assume that there exist an acritical equilibrium web $\mathcal{M}$ and an equilibrum lamination $\mathcal{L}$ for $f$. Then, there exists a subset $\mathcal{P}=\cup_{n} \mathcal{P}_{n} \subset \mathcal{J}$, such that

1. $\operatorname{Card} \mathcal{P}_{n}=d^{n}+o\left(d^{n}\right)$;

2. every $\gamma \in \mathcal{P}_{n}$ is n-periodic; and

3. $\forall M^{\prime} \Subset M$, asymptotically every element of $\mathcal{P}$ is repelling:

$$
\frac{\operatorname{Card}\left\{\gamma \in \mathcal{P}_{n}: \gamma(\lambda) \text { is repelling for every } \lambda \in M^{\prime}\right\}}{\operatorname{Card} \mathcal{P}_{n}} \rightarrow 1 \text {. }
$$

Moreover, $\sum_{\mathcal{P}_{n}} \delta_{\gamma} \rightarrow \mathcal{M}$.

The need to restrict to compact subsets of $M$ is due to the fact that the construction of the equilibrium lamination is essentially local (see Proposition 4.12). Thus, the assumptions of Lemma 4.15 are satisfied on relatively compact subsets of $M$.

Proof We consider the set $\mathcal{P}=\cup_{n} \mathcal{P}_{n} \subset \mathcal{J}$ given by Lemma 4.14. We just need to prove the third assertion. We thus fix $M^{\prime} \Subset M$ and consider the compact metric space $\mathcal{O}\left(M^{\prime}, \overline{\mathcal{U}}, \mathbb{C}^{k}\right)$. By Proposition 4.12 and the implication II. $1 \Rightarrow$ II.3 of Theorem 4.11 all the assumptions of Lemma 4.15 are satisfied by the system $\left(\mathcal{O}\left(M^{\prime}, \overline{\mathcal{U}}, \mathbb{C}^{k}\right), \mathcal{F}, \mathcal{M}\right)$, with $\mathcal{L}$ any equilibrium lamination for the system. The assumption (P2) is verified since this is true at any fixed parameter. The statement follows from the following two assertions:

1. for every repelling periodic $\gamma \in R_{n}$ given by Lemma 4.15, the point $\gamma(\lambda)$ is repelling for every $\lambda \in M^{\prime}$; and

2. asymptotically all elements of $R_{n}$ coincide with elements of $\mathcal{P}_{n}$.

The first point is a consequence of the Lipschitz estimate of the local inverse of $\mathcal{F}^{n}$ at the points of $R_{n}$ (since the Lipschitz constant of $\mathcal{F}^{-n}$ dominates the Lipschitz constant of $f_{\lambda}^{-n}$, for every $\lambda$ ), the second of the fact that both $\mathcal{P}_{n}$ and $R_{n}$ have cardinality $d_{t}^{n}+o\left(d_{t}^{n}\right)$ and, at every $\lambda$, the number of $n$-periodic points is $d_{t}^{n}$. 


\subsection{Proof of Theorem C}

In this section we show that the conditions stated in the Theorems 4.5 and 4.11 are all equivalent. This completes the proof of Theorem C.

Theorem 4.17 Let $f: \mathcal{U} \rightarrow \mathcal{V}$ be a holomorphic family of polynomial-like maps of large topological degree $d_{t} \geq 2$. Assume that the parameter space is simply connected. Then conditions I.1-I.4 of Theorem 4.5 and II.1-II.3 of Theorem 4.11 are all equivalent.

It is immediate to see, by the definition of an equilibrium lamination, that condition II.3 (the existence of a lamination) implies condition I.4 (existence of a graph avoiding the postcritical set), since any element in the lamination satisfies the desired property. Viceversa, by Proposition 4.13, we see that condition I.4 directly implies a local version of Theorem 4.5. Using the uniqueness of the equilibrium lamination, we can nevertheless recover that the conditions in Theorem 4.5 imply the ones in Theorem 4.11 on all the parameter space. This is done in the following proposition.

Proposition 4.18 Let $f: \mathcal{U} \rightarrow \mathcal{V}$ be a holomorphic family of polynomial-like maps of large topological degree $d_{t} \geq 2$. Assume that the parameter space $M$ is simply connected and that every point $\lambda_{0} \in M$ has a neighbourhood where the system admits an equilibrium lamination. Then $f$ admits an equilibrium lamination on all the parameter space.

In particular, if condition I.4 holds, the assumptions of Proposition 4.18 are satisfied (by Proposition 4.13 and Theorem 4.11) and thus condition II.3 holds, too. This complete the proof of Theorem 4.17.

Proof Consider a countable cover $\left\{B_{n}\right\}$ by open balls of the parameter space $M$, with the property that on every $B_{n}$ the system admits an equilibrium lamination $\mathcal{L}_{n}$. In particular, on every $B_{n}$ the restricted system admits an acritical web. Consider two intersecting balls $B_{1}$ and $B_{2}$. By the uniqueness of the equilibrium web on the intersection (which is simply connected), both the corresponding webs induce the same one on $B_{1} \cap B_{2}$. By analytic continuation, and up to removing a zero-measure (for the web on the intersection) subset of graphs from the laminations $\mathcal{L}_{1}$ and $\mathcal{L}_{2}$ (and all their images and preimages, which are always of measure zero), we obtain a set of holomorphic graphs, defined on all of $B_{1} \cup B_{2}$, that satisfy all the properties required in Definition 1.2, thus giving an equilibrium lamination there. The assertion follows repeating the argument, since the cover is countable and $M$ is simply connected (and thus we do not have holonomy problems when glueing the laminations).

Acknowledgements It is a pleasure to thank my advisor François Berteloot for introducing me to this subject and for his patient guidance during the years of my PhD. I would also like to thank Charles Favre, Johan Taflin, and the referee, whose comments greatly helped me to improve both the content and the exposition of this paper. This research was partially supported by the ANR project LAMBDA, ANR13-BS01-0002 and by the FIRB2012 Grant "Differential Geometry and Geometric Function Theory", RBFR12W1AQ 002. 
Open Access This article is distributed under the terms of the Creative Commons Attribution 4.0 International License (http://creativecommons.org/licenses/by/4.0/), which permits unrestricted use, distribution, and reproduction in any medium, provided you give appropriate credit to the original author(s) and the source, provide a link to the Creative Commons license, and indicate if changes were made.

\section{References}

1. Bassanelli, G., Berteloot, F.: Bifurcation currents in holomorphic dynamics on $\mathbb{C P}^{k}$. Journal für die reine und angewandte Mathematik (Crelle's Journal) 2007(608), 201-235 (2007)

2. Berger, P., Dujardin, R.: On stability and hyperbolicity for polynomial automorphisms of $\mathbb{C}^{2}$. Annales Scientifiques de l'École normale supérieure 50(2), 449-477 (2017)

3. Berteloot, F.: Lyapunov exponent of a rational map and multipliers of repelling cycles. Rivista di Matematica della Università di Parma 1(2), 263-269 (2010)

4. Berteloot, F., Bianchi, F.: Perturbations d'exemples de Lattès et dimension de Hausdorff du lieu de bifurcation. To appear on the Journal de Mathématiques Pures et Appliquées, preprint arXiv:1610.08938 (2016)

5. Berteloot, F., Bianchi, F., Dupont, C.: Dynamical stability and Lyapunov exponents for holomorphic endomorphisms of $\mathbb{P}^{k}$. To appear on the Annales Scientifiques de l'École normale supérieure, preprint arXiv:1403.7603 (2015)

6. Bianchi, F.: Motions of Julia sets and dynamical stability in several complex variables. PhD thesis, Université Toulouse III Paul Sabatier and Universitá di Pisa (2016)

7. Bianchi, F., Taflin, J.: Bifurcations in the elementary Desboves family. Proc. AMS 145(10), 4337-4343 (2017)

8. Briend, J.-Y., Duval, J.: Exposants de Liapounoff et distribution des points périodiques d'un endomorphisme de $\mathbb{C P}^{k}$. Acta Math. 182(2), 143-157 (1999)

9. Cornfeld, I.P., Fomin, S.V., Sinai, Y.G.: Ergodic Theory. Springer, New York (1982)

10. DeMarco, L.: Dynamics of rational maps: a current on the bifurcation locus. Math. Res. Lett. 8(1-2), 57-66 (2001)

11. DeMarco, L.: Dynamics of rational maps: Lyapunov exponents, bifurcations, and capacity. Math. Ann. 326(1), 43-73 (2003)

12. Dinh, T.-C., Sibony, N.: Dynamique des applications d'allure polynomiale. Journal de mathématiques pures et appliquées 82(4), 367-423 (2003)

13. Dinh, T.-C., Sibony, N.: Geometry of currents, intersection theory and dynamics of horizontal-like maps. Annales de l'Institut Fourier 56(2), 423-457 (2006)

14. Dinh, T.-C., Sibony, N.: Dynamics in several complex variables: endomorphisms of projective spaces and polynomial-like mappings. In: Holomorphic Dynamical Systems, Springer, New York, pp 165-294 (2010)

15. Dujardin, R.: Non density of stability for holomorphic mappings on $\mathbb{P}^{k}$. Journal de l'École polytechnique 4, 813-843 (2017)

16. Dujardin, R., Lyubich, M.: Stability and bifurcations for dissipative polynomial automorphisms of $\mathbb{C}^{2}$. Invent. Math. 200(2), 439-511 (2013)

17. Federer, H.: Geometric Measure Theory. Springer, New York (1996)

18. Fornaess, J.E., Sibony, N.: Dynamics of $\mathbb{P}^{2}$ (examples). In: Contemporary Mathematics. Laminations and Foliations in Dynamics, Geometry and Topology, vol. 269, pp. 47-86. American Mathematical Society, Providence, RI (2001)

19. Gromov, M.: On the entropy of holomorphic maps. Enseign. Math. 49(3-4), 217-235 (2003)

20. Harvey, R., Shiffman, B.: A characterization of holomorphic chains. Ann. Math. 99(3), 553-587 (1974)

21. John, H.: Hubbard and Peter Papadopol. Superattractive fixed points in $\mathbb{C}^{n}$. Indiana Univ. Math. J. 43(1), 311-365 (1994)

22. Lyubich, M.: Some typical properties of the dynamics of rational maps. Russian Math. Surv. 38(5), 154-155 (1983)

23. Mané, R., Sad, P., Sullivan, D.: On the dynamics of rational maps. Annales scientifiques de l'École Normale Supérieure 16(2), 193-217 (1983)

24. Valery, I.O.: A multiplicative ergodic theorem: characteristic Lyapunov, exponents of dynamical systems. Trudy Moskovskogo Matematicheskogo Obshchestva 19, 179-210 (1968)

25. Parry, W.: Entropy and Generators in Ergodic Theory. WA Benjamin Inc, New York (1969) 
26. Pham, N.-M.: Lyapunov exponents and bifurcation current for polynomial-like maps. Preprint arXiv:math/0512557 (2005)

27. Sibony, N.: Exposé Orsay. (1981)

28. Siu, Y.-T.: Analyticity of sets associated to Lelong numbers and the extension of closed positive currents. Invent. Math. 27(1), 53-156 (1974)

29. Taflin, J.: Blenders near polynomial product maps of $\mathbb{C}^{2}$. Preprint arXiv:1702.02115 (2017) 